\title{
BIFURCATION FOR DIRICHLET PROBLEMS WITHOUT EIGENVALUES
}

\author{
C. A. STUART
}

[Received 11 August 1979-Revised 15 June 1980]

\section{Introduction}

We study the bifurcation of solutions of equations which have one of the following forms:

$$
S u-F(u)=\lambda u
$$

or

$$
S u=\lambda\{u-F(u)\}
$$

where $S$ is a linear self-adjoint operator and $F$ is the gradient of a real-valued function. We suppose that $F(0)=0$ and that $F$ is of order higher than linear at the origin. The classical results deal with bifurcation from isolated eigenvalues, $\lambda$, of finite multiplicity of $S$. At such points, $S-\lambda I$ is a Fredholm operator and the method of Lyapunov and Schmidt reduces the problem to the study of an equation in the null space of $S-\lambda I$. In this paper we are especially interested in bifurcation from points $\lambda$ at which $S-\lambda I$ is not a Fredholm operator. In particular, the case where $S-\lambda I$ is injective but not surjective occurs naturally in the $L^{2}$-theory of elliptic equations on unbounded domains. For such problems the Lyapunov-Schmidt reduction cannot be used and we must work directly with the infinite-dimensional problem.

In $\S 2$ we introduce the basic notation and assumptions adopted throughout the paper. The special hypotheses used to establish bifurcation for the equations (1.1) and (1.2) are given in $\S \S 3$ and 4 . The remainder of the paper is devoted to applications of these general results to the following two non-linear eigenvalue problems. Find $u \in L^{2}\left(\mathbb{R}^{n}\right)$ and $\lambda \in \mathbb{R}$ such that

$$
-\Delta u(x)-r(x)|u(x)|^{\sigma} u(x)=\lambda u(x) \text { for } x \in \mathbb{R}^{n},
$$

or

$$
-\Delta u(x)=\lambda\left\{u(x)-r(x)|u(x)|^{\sigma} u(x)\right\} \quad \text { for } x \in \mathbb{R}^{n},
$$

where the function $r: \mathbb{R}^{n} \rightarrow[0, \infty)$ and the constant $\sigma>0$ are given. For such problems, one of the requirements of the general theory is that the functional,

$$
\varphi(u)=\frac{1}{(\sigma+2)} \int_{\mathbb{R}^{n}} r(x)|u(x)|^{\sigma+2} d x,
$$

be weakly sequentially continuous on the Sobolev space

$$
W_{2}^{1}\left(\mathbb{R}^{n}\right) \equiv\left\{u \in L^{2}\left(\mathbb{R}^{n}\right): \partial_{i} u \in L^{2}\left(\mathbb{R}^{n}\right) \text { for } 1 \leqslant i \leqslant n\right\} .
$$

(Here $\partial_{i} u$ denotes the $i$ th partial derivative of $u$ in the sense of distributions.) In $\S 5$ we Proc. London Math. Soc. (3), 45 (1982), 169-192. 
ensure this by requiring that $r(x) \rightarrow 0$ as $|x| \rightarrow \infty$ (Lemma 5.2). If $r$ is merely bounded, $\varphi$ is not necessarily weakly sequentially continuous. However, for $n \geqslant 2$, this behaviour can be recovered by restricting attention to the radially symmetric elements of $W_{2}^{1}\left(\mathbb{R}^{n}\right)$, provided that $r$ is radially symmetric. This device, which allows us to cover the case where $r$ is constant, is used in $\$ 6$.

In the one-dimensional case, the weak sequential continuity of $\varphi$ is not recovered by restricting attention to even elements of $W_{2}^{1}(\mathbb{R})$. In this case $(1.3)$ is equivalent to the Neumann problem,

$$
\begin{aligned}
& -u^{\prime \prime}(x)-r(x)|u(x)|^{\sigma} u(x)=\lambda u(x) \text { for } x>0, \\
& u^{\prime}(0)=0, \quad u \in L^{2}(0, \infty),
\end{aligned}
$$

provided that $r$ is even. The persistent lack of weak sequential continuity means that the problem (1.5) requires special attention $[4,12]$.

In $\S 7$, we discuss the corresponding Dirichlet problem,

$$
\begin{aligned}
& -w^{\prime \prime}(x)-r(x)|w(x)|^{\sigma} w(x)=\lambda w(x) \text { for } x>0, \\
& w(0)=0, \quad w \in L^{2}(0, \infty),
\end{aligned}
$$

where the function $r:(0, \infty) \rightarrow[0, \infty)$ is bounded and measurable on compact subsets of $(0, \infty)$. This problem arises if we seek radially symmetric solutions of $(1.3)$ in the case where $n=3[10,11]$, but it is also of independent interest.

For the problems (1.3) and (1.4), $\lambda=0$ is the infimum of the spectrum of the linearized problem,

$$
\begin{aligned}
& -\Delta u(x)=\lambda u(x) \text { for } x \in \mathbb{R}^{n}, \\
& u \in L^{2}\left(\mathbb{R}^{n}\right),
\end{aligned}
$$

and the results in $\S \S 3$ and 4 furnish conditions on $\sigma>0$ and the function $r$ which imply that 0 is a bifurcation point for the non-linear problems. These conditions depend upon the measure of the size of a solution with respect to which bifurcation is considered. Here we concentrate on bifurcation in terms of the measures

$$
\int_{\mathbf{R}^{n}} u(x)^{2} d x \text { and } \int_{\mathbf{R}^{n}}|\nabla u(x)|^{2} d x .
$$

Elsewhere $[10,11,13]$ we have considered bifurcation with respect to the quantities

$$
\int_{\mathbf{R}^{n}}\left\{u(x)^{2}+|\nabla u(x)|^{2}\right\} d x \text { and } \int_{R^{n}}\left\{u(x)^{2}+[\Delta u(x)]^{2}\right\} d x,
$$

and we have treated more general non-linearities.

As in the related articles [9-13], our results give conditions under which there is bifurcation to the left of the lowest point of the spectrum of the linearization. Complementary results, dealing with bifurcation to the right of the lowest point of the spectrum, have appeared recently [1].

The relationship between equations (1.3) and (1.4) and some problems from physics is discussed in $[7,8]$.

\section{General assumptions}

Let $H$ denote a real Hilbert space with norm $\|\cdot\|$ and scalar product $\langle\cdot, \cdot\rangle$. If $L: \mathscr{D}(L) \subset H \rightarrow H$ is a linear operator whose domain, $\mathscr{D}(L)$, is a dense subspace of $H$, 
then its adjoint in $H[14]$ is denoted by $L^{*}: \mathscr{D}\left(L^{*}\right) \subset H \rightarrow H$. We suppose henceforth that the following condition holds.

(C0) Let $S: \mathscr{D}(S) \subset H \rightarrow H$ be a positive self-adjoint operator.

That is to say, $S=S^{*}$ and $\langle S u, u\rangle \geqslant 0$ for all $u \in \mathscr{D}(S)$. In particular, $S$ is a closed operator and its domain, $\mathscr{D}(S)$, equipped with the graph norm of $S$,

$$
\|u\|_{2}=\left\{\|u\|^{2}+\|S u\|^{2}\right\}^{\frac{1}{2}},
$$

is a Hilbert space which will be denoted by $H_{2}$. Let $T: \mathscr{D}(T) \subset H \rightarrow H$ be a closed operator which is densely defined and such that $S=T^{*} T$. (For example, we could choose $T$ to be the positive self-adjoint square root of $S$, but this is not always the most convenient choice [9-12].) The domain of $T$ equipped with the graph norm of $T$,

$$
\|u\|_{1}=\left\{\|u\|^{2}+\|T u\|^{2}\right\}^{\frac{1}{2}},
$$

is a Hilbert space which we denote by $H_{1}$. Then $H_{2} \subset H_{1} \subset H$ and these embeddings are continuous. Furthermore, $T$ can be regarded as a bounded linear operator from $H_{1}$ into $H$ and, as such, it will be denoted by $T_{1}: H_{1} \rightarrow H$. Then the dual (or conjugate) operator $T_{1}^{\prime}: H^{*} \rightarrow H_{1}^{*}$ is also bounded [14]. Now $\mathscr{D}(T)$ is a dense subset of $H$ and so $H^{*}$ can be simultanously identified with $H$ and with a subspace of $H_{1}^{*}$. Thus we can write $H_{1} \subset H=H^{*} \subset H_{1}^{*}$ and this identification is such that we can write $f(v)=\langle f, v\rangle$ whenever

$$
f \in H^{*} \text { and } v \in H \text { or } f \in H_{1}^{*} \text { and } v \in H_{1} .
$$

With these identifications, it is easy to see that

$$
\begin{aligned}
& T_{1}^{\prime} \text { is an extension of } T^{*}, \\
& \mathscr{D}\left(T^{*}\right)=\left\{v \in H: T_{1}^{\prime} v \in H\right\}, \\
& T_{1}^{\prime} T_{1}: H^{1} \rightarrow H_{1}^{*} \text { is an extension of } S, \\
& H_{2}=\mathscr{D}(S)=\left\{v \in H_{1}: T_{1}^{\prime} T_{1} v \in H\right\},
\end{aligned}
$$

and

$$
\langle T u, T v\rangle=\left\langle T_{1}^{\prime} T_{1} u, v\right\rangle=\left\langle T_{1}^{\prime} T_{1} v, u\right\rangle,
$$

for all $u, v \in H_{1}$. (These identifications and their consequences are dealt with in detail in [13].)

We can now formulate our basic assumption about the form of the mapping $F$.

(C1) Let $\varphi: H_{1} \rightarrow \mathbb{R}$ be a continuously Fréchet differentiable mapping such that $\varphi(0)=0$. Then, for each $u \in H_{1}, \varphi^{\prime}(u) \in H_{1}^{*}$ and so we can write

$$
\varphi^{\prime}(u) v=\langle F(u), v\rangle \text { for all } u, v \in H_{1},
$$

where $F: H_{1} \rightarrow H_{1}^{*}$ is a continuous mapping.

When the condition (C1) holds, we say that $F$ is the gradient of $\varphi$ and that $\varphi$ is a potential for $F$. We note that the operator $T_{1}^{\prime} T_{1}: H_{1} \rightarrow H_{1}^{*}$ is a gradient and that $\frac{1}{2}\left\|T_{1} u\right\|^{2}$ is a potential for $T_{1}^{\prime} T_{1}$. 


\section{Bifurcation for the problem (1.1)}

In this section we give results concerning the bifurcation of solutions of equations of the form

$$
S u-F(u)=\lambda u \text {. }
$$

We use the general notation and assumptions introduced in $\S 2$. In particular, the conditions $(\mathrm{C} 0)$ and $(\mathrm{C} 1)$ are supposed to hold.

We say that $(u, \lambda) \in H \times \mathbb{R}$ is a generalized solution of (1.1) if

$$
u \in H_{1} \quad \text { and } \quad T_{1}^{\prime} T_{1} u-F(u)=\lambda u .
$$

If, in addition,

$$
u \in H_{2} \text { and } F(u) \in H
$$

then $(u, \lambda)$ is called a strong solution of $(3.1)$.

We have previously [9-11] discussed (3.1) under the following stronger assumption.

(C1)' The condition (C1) holds and $F$ maps $H_{1}$ continuously into $H$.

Our terminology is justified by the observation that if the conditions $(\mathrm{C} 0)$ and $(\mathrm{C} 1)^{\prime}$ are satisfied and $(u, \lambda)$ is a generalized solution of $(3.1)$, then $(u, \lambda)$ is in fact a strong solution.

Suppose that the conditions (C0) and (C1) hold and that $F(0)=0$. Let

$$
E G=\left\{(u, \lambda) \in H_{1} \times \mathbb{R}: T_{1}^{\prime} T_{1} u-F(u)=\lambda u \text { and } u \neq 0\right\}
$$

and

$$
E S=\left\{(u, \lambda) \in E G: u \in H_{2} \text { and } F(u) \in H\right\} .
$$

The notion of bifurcation depends upon the quantity used to measure the size of a solution. The following two cases seem natural. We write $\lambda_{0} \in B G$ if and only if there exists a sequence $\left\{\left(u_{k}, \lambda_{k}\right)\right\}$ in $E G$ such that $\lambda_{k} \rightarrow \lambda_{0}$ and $\left\|u_{k}\right\| \rightarrow 0$ as $k \rightarrow \infty$. (We allow the possibility that $\pm \infty \in B G$.) We write $\lambda_{0} \in B G^{*}$ if and only if there exists a sequence $\left\{\left(u_{k}, \lambda_{k}\right)\right\}$ in $E G$ such that $\lambda_{k} \rightarrow \lambda_{0}$ and $\left\|T_{1} u_{k}\right\| \rightarrow 0$ as $k \rightarrow \infty$.

Similarly, when dealing with bifurcation from infinity we use the notation $\lambda_{0} \in B G(\infty) / B G^{*}(\infty)$ if and only if there exists a sequence $\left\{\left(u_{k}, \lambda_{k}\right)\right\}$ in $E G$ such that $\lambda_{k} \rightarrow \lambda_{0}$ as $\left\|u_{k}\right\| \rightarrow \infty /\left\|T_{1} u_{k}\right\| \rightarrow \infty$ as $k \rightarrow \infty$.

The following assumptions allow us to study bifurcation by a simple variational argument.

(C2) For all $t \geqslant 1$ and all $u \in H_{1}, \varphi(t u) \geqslant t^{2} \varphi(u) \geqslant 0$ and $\langle F(u), u\rangle \geqslant 2 \varphi(u)$.

(C3) There exist constants $K>0, \alpha \in[0,2)$, and $\beta>0$ such that $\alpha+\beta>2$ and

$$
|\langle F(u), u\rangle| \leqslant K\|T u\|^{\alpha}\|u\|^{\beta} \quad \text { for all } u \in H_{1} .
$$

Note that (C1) and (C3) together imply that $F(0)=0$, and, if $F: H_{1} \rightarrow H_{1}^{*}$ is continuously differentiable at 0 , that $F^{\prime}(0)=0[10,11]$.

Let $\psi: H_{1} \rightarrow \mathbb{R}$ be defined by $\psi(u)=\frac{1}{2}\left\|T_{1} u\right\|^{2}-\varphi(u)$. For $c>0$, let $S(c)=\left\{u \in H_{1}:\|u\|=c\right\}$ and $m(c)=\inf \{\psi(u): u \in S(c)\}$. Note that $S(c)$ is an unbounded subset of $H_{1}$ (unless $S$ is a bounded operator in $H$ ). 
Lemma 3.1. Let the conditions (C0), (C1), and (C3) hold. Then $B G \subset[0, \infty) \cup\{+\infty\}$ for equation (3.1).

Proof. Suppose that $\left\{\left(u_{k}, \lambda_{k}\right)\right\}$ is a sequence in $E G$ such that $\lim _{k \rightarrow \infty}\left\|u_{k}\right\|=0$ and $\lambda_{k}<0$ for all $k \in \mathbb{N}$. Then, for all $k \in \mathbb{N}$,

$$
\left\langle T u_{k}, T u_{k}\right\rangle-\left\langle F\left(u_{k}\right), u_{k}\right\rangle=\lambda_{k}\left\|u_{k}\right\|^{2}
$$

and so, by (C3), $-K\left\|T u_{k}\right\|^{\alpha}\left\|u_{k}\right\|^{\beta} \leqslant \lambda_{k}\left\|u_{k}\right\|^{2}$. On the other hand, since $\lambda_{k}<0$,

$$
\left\|T u_{k}\right\|^{2} \leqslant K\left\|T u_{k}\right\|^{\alpha}\left\|u_{k}\right\|^{\beta} .
$$

Hence

$$
\begin{aligned}
\lambda_{k}\left\|u_{k}\right\|^{2} & \geqslant-K\left[K\left\|u_{k}\right\|^{\beta}\right]^{\alpha /(2-\alpha)}\left\|u_{k}\right\|^{\beta} \\
& =-K^{2 /(2-\alpha)}\left\|u_{k}\right\|^{2 \beta /(2-\alpha)},
\end{aligned}
$$

and so $\lambda_{k} \geqslant-K^{2 /(2-\alpha)}\left\|u_{k}\right\|^{2(\alpha+\beta-2) /(2-\alpha)}$ since $\left\|u_{k}\right\| \neq 0$. Since $\alpha+\beta-2>0$ and $2-\alpha>0$, we thus have that $\liminf _{k \rightarrow \infty} \lambda_{k} \geqslant 0$. This proves that $B G \subset[0, \infty) \cup\{+\infty\}$.

Proposition 3.2. Let the conditions (C0) to (C3) hold. Suppose in addition that

(i) $\varphi: H_{1} \rightarrow \mathbb{R}$ is weakly sequentially continuous $\left(\right.$ i.e. $\varphi\left(u_{n}\right) \rightarrow \varphi(u)$ whenever $u_{n}$ converges weakly to $u$ in $H_{1}$ ),

(ii) $m(c)<0$.

Then $m(c)>-\infty$ and there exists $\left(u_{c}, \lambda_{c}\right) \in H_{1} \times \mathbb{R}$ such that

$$
\begin{gathered}
\left\|u_{c}\right\|=c, \quad\left\|T u_{c}\right\| \leqslant\left[K c^{\beta}\right]^{1 /(2-\alpha)}, \quad \psi\left(u_{c}\right)=m(c), \\
-K^{2 /(2-\alpha)} c^{2(\alpha+\beta-2) /(2-\alpha)} \leqslant \lambda_{c} \leqslant 2 m(c) / c^{2}<0,
\end{gathered}
$$

and $T_{1}^{\prime} T_{1} u_{c}-F\left(u_{c}\right)=\lambda_{c} u_{c}$ in $H_{1}^{*}$.

Proof. Let $\left\{\left(u_{k}, \lambda_{k}\right)\right\}$ be a sequence in $S(c)$ such that $\psi\left(u_{k}\right) \rightarrow m(c)$ as $k \rightarrow \infty$. We may suppose that $\psi\left(u_{k}\right)<0$ for all $k \in \mathbb{N}$ and hence, using (C2) and (C3), we have that

$$
\left\|T u_{k}\right\|^{2}<2 \varphi\left(u_{k}\right) \leqslant\left\langle F\left(u_{k}\right), u_{k}\right\rangle \leqslant K\left\|T u_{k}\right\|^{\alpha}\left\|u_{k}\right\|^{\beta} \text {. }
$$

Thus we see that $\left\|T u_{k}\right\| \leqslant\left[K\left\|u_{k}\right\|^{\beta}\right]^{1 /(2-\alpha)}=\left[K c^{\beta}\right]^{1 /(2-\alpha)}$ for all $k \in \mathbb{N}$, since $\alpha \in[0,2)$. This implies that the minimizing sequence $\left\{u_{k}\right\}$ is bounded in $H_{1}$. By passing to a subsequence, we may suppose that $u_{k} \rightarrow u_{c}$ weakly in $H_{1}$. That is,

$$
\left\langle w, u_{k}-u_{c}\right\rangle \rightarrow 0 \text { as } k \rightarrow \infty \text { for all } w \in H_{1}^{*} .
$$

In particular, $u_{k} \rightarrow u_{c}$ weakly in $H$ since $H=H^{*} \subset H_{1}^{*}$. This implies that $\left\|u_{c}\right\| \leqslant \liminf _{k \rightarrow \infty}\left\|u_{k}\right\|=c$. Furthermore, for $v \in H$,

$$
\left\langle v, T_{1}\left(u_{k}-u_{c}\right)\right\rangle=\left\langle T_{1}^{\prime} v, u_{k}-u_{c}\right\rangle \rightarrow 0
$$

as $k \rightarrow \infty$ and so $T_{1} u_{k} \rightarrow T_{1} u_{c}$ weakly in $H$. This implies that

$$
\left\|T_{1} u_{c}\right\| \leqslant \liminf _{k \rightarrow \infty}\left\|T_{1} u_{k}\right\| \text {. }
$$

From (i), it now follows that

$$
\psi\left(u_{c}\right)=\frac{1}{2}\left\|T_{1} u_{c}\right\|^{2}-\varphi\left(u_{c}\right) \leqslant \liminf _{k \rightarrow \infty}\left\{\frac{1}{2}\left\|T_{1} u_{k}\right\|^{2}-\varphi\left(u_{k}\right)\right\}=m(c) .
$$


Since $m(c)<0$ and $\varphi(0)=0$, we have that $u_{c} \neq 0$. Setting $t=c /\left\|u_{c}\right\|$, we have that $1 \leqslant t<\infty$. But

$$
\begin{aligned}
\psi\left(t u_{c}\right) & =\frac{1}{2} t^{2}\left\|T u_{c}\right\|^{2}-\varphi\left(t u_{c}\right) \leqslant t^{2} \psi\left(u_{c}\right) \quad(\text { by }(\mathrm{C} 2)) \\
& \leqslant t^{2} m(c) \quad\left(\text { since } t^{2} \geqslant 1 \text { and } \psi\left(u_{c}\right) \leqslant m(c)\right) \\
& \leqslant t^{2} \psi\left(t u_{c}\right) \quad\left(\text { since } t u_{c} \in S(c)\right) .
\end{aligned}
$$

Hence $\left(t^{2}-1\right) \psi\left(t u_{c}\right) \geqslant 0$ and $\psi\left(t u_{c}\right) \leqslant t^{2} m(c)<0$. This means that $t^{2} \leqslant 1$. Since we already have that $t \geqslant 1$, we conclude that $t=1$. Thus we see that $u_{c} \in S(c)$ and that $\psi\left(u_{c}\right)=m(c)$.

It now follows from the Euler-Lagrange principle [13] that there exists $\lambda_{c} \in \mathbb{R}$ such that $T_{1}^{\prime} T_{1} u_{c}-F\left(u_{c}\right)=\lambda_{c} u_{c}$. Since $\psi\left(u_{c}\right)=m(c)<0$, it follows that

$$
\left\|T u_{c}\right\| \leqslant\left[K c^{\beta}\right]^{1 /(2-\alpha)} \text {. }
$$

Furthermore,

$$
\lambda_{c}\left\|u_{c}\right\|^{2}=\left\|T u_{c}\right\|^{2}-\left\langle F\left(u_{c}\right), u_{c}\right\rangle \leqslant 2 \psi\left(u_{c}\right)=2 m(c), \quad \text { by }(\mathrm{C} 2),
$$

and so

$$
\lambda_{c} \leqslant 2 m(c) / c^{2}<0
$$

On the other hand, as in the proof of Lemma 3.1, we have that

$$
\lambda_{c} \geqslant-K^{2 /(2-\alpha)} c^{2(\alpha+\beta-2) /(2-\alpha)} \text {. }
$$

This completes the proof of the proposition.

THEOREM 3.3. Let the conditions (C0) to (C3) hold and suppose also that $\varphi: H_{1} \rightarrow \mathbb{R}$ is weakly sequentially continuous.

(a) If there exists $c_{0}>0$ such that $m(c)<0$ for all $c \in\left(0, c_{0}\right)$, then $0 \in B G$ for equation (3.1).

(b) If $m(c) / c^{2} \rightarrow-\infty$ as $c \rightarrow+\infty$, then $-\infty \in B G(\infty)$ for equation (3.1).

Proof. These results follow immediately from Proposition 3.2.

In dealing with bifurcation with respect to $\left\|T_{1} u\right\|$, it is appropriate to replace (C3) by the following condition.

(C3)* There exist constants $K>0, \alpha>0, \beta \in[0,2)$ such that $\alpha+\beta>2$ and

$$
|\langle F(u), u\rangle| \leqslant K\|T u\|^{\alpha}\|u\|^{\beta} \text { for all } u \in H_{1} .
$$

As before $(\mathrm{C} 1)$ and $(\mathrm{C} 3)^{*}$ together imply that $F(0)=0$. Let $\psi^{*}: H_{1} \rightarrow \mathbb{R}$ be defined by $\psi^{*}(u)=\frac{1}{2}\|u\|^{2}-\varphi(u)$. For $c>0$, set $S^{*}(c)=\left\{u \in H_{1}:\|T u\|=c\right\}$ and $M(c)=\inf \left\{\psi^{*}(c): u \in S^{*}(c)\right\}$. Note that $S^{*}(c)$ is an unbounded subset of $H_{1}$ (unless $S$ has a bounded inverse in $H$ ).

Lemma 3.4. Let the conditions (C0), (C1), and (C3)* hold. Then

$$
B G^{*} \subset[0, \infty) \cup\{+\infty\}
$$

for equation (3.1). 
Proof. Suppose that $\left\{\left(u_{k}, \lambda_{k}\right)\right\}$ is a sequence in $E G$ such that $\lim _{k \rightarrow \infty}\left\|T_{1} u_{k}\right\|=0$ and $\lambda_{k}<0$ for all $k \in \mathbb{N}$. Then

$$
\left\|T u_{k}\right\|^{2}-\left\langle F\left(u_{k}\right), u_{k}\right\rangle=\lambda_{k}\left\|u_{k}\right\|^{2} \quad \text { for all } k \in \mathbb{N} .
$$

Hence

$$
-K\left\|T u_{k}\right\|^{\alpha}\left\|u_{k}\right\|^{\beta} \leqslant \lambda_{k}\left\|u_{k}\right\|^{2}
$$

and

$$
\left\|T u_{k}\right\|^{2} \leqslant K\left\|T u_{k}\right\|^{\alpha}\left\|u_{k}\right\|^{\beta} .
$$

Since $u_{k} \neq 0$ and $\lambda_{k}<0$, it follows that $\left\|T u_{k}\right\| \neq 0$. If $\beta>0$, we have that

$$
\begin{aligned}
\left|\lambda_{k}\right| & \leqslant K\left\|T u_{k}\right\|^{\alpha}\left\|u_{k}\right\|^{\beta-2} \\
& \leqslant K\left\|T u_{k}\right\|^{\alpha}\left\{K\left\|T u_{k}\right\|^{(\alpha-2))(2-\beta) / \beta}\right. \\
& =K^{2 / \beta}\left\|T_{1} u_{k}\right\|^{2(\alpha+\beta-2) / \beta} \quad \text { for all } k \in \mathbb{N} .
\end{aligned}
$$

This proves that $\lim _{k \rightarrow \infty}\left|\lambda_{k}\right|=0$ and so $B G^{*} \subset[0, \infty) \cup\{+\infty\}$ if $\beta>0$. If $\beta=0$, $\left\|T u_{k}\right\|^{2} \leqslant K\left\|T u_{k}\right\|^{\alpha}$ where $\alpha>2$ and so $\left\|T_{1} u_{k}\right\| \geqslant K^{1 /(2-\alpha)}$. But $\left\|T u_{k}\right\| \rightarrow 0$ as $k \rightarrow \infty$. Hence $B G^{*} \subset[0, \infty) \cup\{+\infty\}$ if $\beta=0$.

We can deduce a sufficient condition for bifurcation with respect to $\left\|T_{1} u\right\|$ from the results proved in $\S 4$ provided that $F$ is homogeneous of degree $\sigma+1$ for some $\sigma>0$, that is,

$$
F(t u)=t^{\sigma+1} F(u) \text { for all } t>0 \text { and all } u \in H_{1} .
$$

THEOREM 3.5. Let the conditions (C0), (C1), (C2), and (C3)* hold. Suppose also that $\varphi: H_{1} \rightarrow \mathbb{R}$ is weakly sequentially continuous and that $F$ is homogeneous of degree $\sigma+1$ where $\sigma>0$.

(a) If $M(c) / c^{2+\sigma} \rightarrow-\infty$ as $c \rightarrow+\infty$, then $0 \in B G^{*}$ for equation (3.1).

(b) If $\beta>0$ and there exists $c_{0}>0$ such that $M(c)<0$ for all $c \in\left(0, c_{0}\right)$, then $-\infty \in B G^{*}(\infty)$ for equation (3.1).

Proof. This follows immediately from Proposition 4.4 and the observation that, for $\lambda<0,(u, \lambda)$ is a generalized solution of equation (4.1) if and only if $\left(|\lambda|^{1 / \sigma} u, \lambda\right)$ is a generalized solution of equation (3.1).

REMARKS. 1. We have chosen to stress the results obtained for bifurcation with respect to $\|u\|$ and $\left\|T_{1} u\right\|=\left\|S^{\frac{1}{2}} u\right\|$, but Proposition 3.2 also yields information about bifurcation with respect to other measures of the size of a solution. For example, under the hypotheses of Theorem 3.3 (a), 0 is a bifurcation point with respect to

$$
\|u\|_{1}=\left\{\|u\|^{2}+\|T u\|^{2}\right\}^{\frac{1}{2}} .
$$

Furthermore, if $(\mathrm{C} 1)$ is replaced by the stronger assumption $(\mathrm{C} 1)^{\prime}$ then we have bifurcation of strong solutions with respect to the norm

$$
\|u\|_{2}=\left\{\|u\|^{2}+\|S u\|^{2}\right\}^{\frac{1}{2}},
$$

under the same hypotheses. (See [13] for details.) 
2. Under the hypotheses of Theorems 3.3 (a) or 3.5 (a) we have that $0=\inf \sigma(S)$. (See [10] for details.)

\section{Bifurcation for the problem (1.2)}

In this section we deal with equations which have the form

$$
S u=\lambda\{u-F(u)\},
$$

where we again adopt the general hypotheses and conventions set out in $\S 2$. We use the notation introduced in $\S 3$ for the sets of bifurcation points for the equation (4.1), except that in the present context,

$$
E G \equiv\left\{(u, \lambda) \in H_{1} \times \mathbb{R}: T_{1}^{\prime} T_{1} u=\lambda[u-F(u)] \text { and } u \neq 0\right\}
$$

is the set of all generalized solutions of (4.1) and

$$
E S \equiv\left\{(u, \lambda) \in E G: u \in H_{2} \text { and } F(u) \in H\right\}
$$

is the set of strong solutions of (4.1).

LEMma 4.1. Let the conditions (C0), (C1), and (C3) hold. Then

$$
B G \subset[0, \infty) \cup\{ \pm \infty\}
$$

for equation (4.1).

Proof. Suppose that $\left\{\left(u_{k}, \lambda_{k}\right)\right\}$ is a sequence in $E G$ such that $\lim _{k \rightarrow \infty}\left\|u_{k}\right\|=0$ and $\lambda_{k}<0$ for all $k \in \mathbb{N}$. Then

$$
\left\|T u_{k}\right\|^{2}=\lambda_{k}\left\{\left\|u_{k}\right\|^{2}-\left\langle F\left(u_{k}\right), u_{k}\right\rangle\right\}
$$

and so

$$
\lambda_{k}^{-1}\left\|T u_{k}\right\|^{2} \geqslant-K\left\|T u_{k}\right\|^{\alpha}\left\|u_{k}\right\|^{\beta}
$$

and

$$
\left\|u_{k}\right\|^{2} \leqslant K\left\|T u_{k}\right\|^{\alpha}\left\|u_{k}\right\|^{\beta} \quad \text { for all } k \in \mathbb{N}
$$

Hence, if $\alpha>0$, we have $\left\|T u_{k}\right\| \neq 0$ and

$$
\begin{aligned}
\lambda_{k}^{-1} & \geqslant-K\left\|T u_{k}\right\|^{\alpha-2}\left\|u_{k}\right\|^{\beta} \\
& \geqslant-K\left[K^{-1 / \alpha}\left\|u_{k}\right\|^{(2-\beta) / \alpha}\right]^{\alpha-2}\left\|u_{k}\right\|^{\beta} \\
& =-K^{2 / \alpha}\left\|u_{k}\right\|^{2(\alpha+\beta-2) / \alpha} .
\end{aligned}
$$

This proves that $\lambda_{k} \rightarrow-\infty$ if $\alpha>0$. If $\alpha=0$, then $\left\|u_{k}\right\|^{2} \leqslant K\left\|u_{k}\right\|^{\beta}$ where $\beta>2$, and consequently $\left\|u_{k}\right\| \geqslant K^{1 /(2-\beta)}$. This proves the lemma.

THEOREM 4.2. Let the conditions (C0) to (C3) hold. Suppose also that $\varphi: H_{1} \rightarrow \mathbb{R}$ is weakly sequentially continuous and that $F$ is homogeneous of degree $\sigma+1$ where $\sigma>0$.

(a) If $m(c) / c^{2+\sigma} \rightarrow-\infty$ as $c \rightarrow \infty$, then $-\infty \in B G$ for equation (4.1).

(b) If $\alpha>0$ and there exists $c_{0}>0$ such that $m(c)<0$ for all $c \in\left(0, c_{0}\right)$, then $0 \in B G(\infty)$ for equation (4.1). 
Proof. This follows immediately from Proposition 3.2 and the observation that, for $\lambda<0,(u, \lambda) \in E G$ for $(4.1)$ if and only if $\left(|\lambda|^{1 / \sigma} u, \lambda\right) \in E G$ for (3.1).

Lemma 4.3. Let the conditions $(\mathrm{C} 0),(\mathrm{C} 1)$, and $(\mathrm{C} 3)^{*}$ hold. Then

$$
B G^{*} \subset[0, \infty) \cup\{ \pm \infty\}
$$

for equation (4.1).

Proof. Let $\left\{\left(u_{k}, \lambda_{k}\right)\right\}$ be a sequence in $E G$ such that $\lim _{k \rightarrow \infty}\left\|T_{1} u_{k}\right\|=0$ and $\lambda_{k}<0$ for all $k \in \mathbb{N}$. Then

$$
\left\|T u_{k}\right\|^{2}=\lambda_{k}\left\{\left\|u_{k}\right\|^{2}-\left\langle F\left(u_{k}\right), u_{k}\right\rangle\right\}
$$

and so

$$
\lambda_{k}^{-1}\left\|T u_{k}\right\|^{2} \geqslant-K\left\|T u_{k}\right\|^{\alpha}\left\|u_{k}\right\|^{\beta}
$$

and

$$
\left\|u_{k}\right\|^{2} \leqslant K\left\|T u_{k}\right\|^{\alpha}\left\|u_{k}\right\|^{\beta} \quad \text { for all } k \in \mathbb{N} .
$$

Combining these inequalities we obtain

$$
\left\|T u_{k}\right\| \neq 0 \text { and } 0>\lambda_{k}^{-1} \geqslant-K^{2 /(2-\beta)}\left\|T u_{k}\right\|^{2(\alpha+\beta-2) /(2-\beta)} .
$$

This proves the result.

Proposition 4.4. Let the conditions (C0), (C1), (C2), and (C3)* hold. Suppose in addition that

(i) $\varphi: H_{1} \rightarrow \mathbb{B}$ is weakly sequentially continuous,

(ii) $M(c)<0$.

Then $M(c)>-\infty$ and there exists $\left(u_{c}, \lambda_{c}\right) \in H_{1} \times \mathbb{R}$ such that

$$
\begin{gathered}
\left\|T u_{c}\right\|=c, \quad\left\|u_{c}\right\| \leqslant\left[K c^{\alpha}\right]^{1 /(2-\beta)}, \quad \psi^{*}\left(u_{c}\right)=M(c), \\
c^{2} / 2 M(c) \leqslant \lambda_{c} \leqslant-K^{2 /(\beta-2)} c^{2(\alpha+\beta-2) /(\beta-2)}
\end{gathered}
$$

and

$$
T_{1}^{\prime} T_{1} u_{c}=\lambda_{c}\left\{u_{c}-F\left(u_{c}\right)\right\} \quad \text { in } H_{1}^{*}
$$

Proof. Let $\left\{\left(u_{k}, \lambda_{k}\right)\right\}$ be a sequence in $S^{*}(c)$ such that $\psi^{*}\left(u_{k}\right) \rightarrow M(c)$ as $k \rightarrow \infty$. We may suppose that $\psi^{*}\left(u_{k}\right)<0$ for all $k \in \mathbb{N}$. Hence, using $(\mathrm{C} 2)$ and $(\mathrm{C} 3)^{*}$, we have that

$$
\left\|u_{k}\right\| \leqslant\left[K\left\|T u_{k}\right\|^{\alpha}\right]^{1 /(2-\beta)}=\left[K c^{\alpha}\right]^{1 /(2-\beta)},
$$

for all $k \in \mathbb{N}$. This implies that the sequence $\left\{u_{k}\right\}$ is bounded in $H_{1}$. Hence, as in the proof of Proposition 3.2, there exists $u_{c} \in H_{1}$ such that $u_{k} \rightarrow u_{c}$ weakly in $H$ (and in $H_{1}$ ) and $T u_{k} \rightarrow T u_{c}$ weakly in $H$. In particular,

and

$$
\left\|T u_{c}\right\| \leqslant \liminf _{k \rightarrow \infty}\left\|T u_{k}\right\|=c
$$

$$
\psi^{*}\left(u_{c}\right)=\frac{1}{2}\left\|u_{c}\right\|^{2}-\varphi\left(u_{c}\right) \leqslant \liminf _{k \rightarrow \infty}\left\{\frac{1}{2}\left\|u_{k}\right\|^{2}-\varphi\left(u_{k}\right)\right\}=M(c) .
$$

Since $M(c)<0$, we see that $\varphi\left(u_{c}\right)>0$. But according to (C2) and (C3)*,

$$
0 \leqslant 2 \varphi\left(u_{c}\right) \leqslant K\left\|T u_{c}\right\|^{\alpha}\left\|u_{c}\right\|^{\beta} \quad \text { where } \alpha>0 .
$$


This shows that $T u_{c} \neq 0$. Setting $t=c /\left\|T u_{c}\right\|$, we have that $t \geqslant 1$. Then, arguing as in the proof of Proposition 3.2, we find that $t=1$. Hence $u_{c} \in S^{*}(c), \psi^{*}\left(u_{c}\right)=M(c)$, and there exists $\xi_{c} \in \mathbb{R}$ such that

$$
u_{c}-F\left(u_{c}\right)=\xi_{c} T_{1}^{\prime} T_{1} u_{c} \text { in } H_{1}^{*} .
$$

Since $\psi^{*}\left(u_{c}\right)=M(c)<0$, it follows that $\left\|u_{c}\right\| \leqslant\left[K c^{\alpha}\right]^{1 / 2-\beta)}$. Furthermore,

$$
\begin{aligned}
\xi_{c}\left\|T u_{c}\right\|^{2} & =\left\|u_{c}\right\|^{2}-\left\langle F\left(u_{c}\right), u_{c}\right\rangle \\
& \leqslant 2 \psi^{*}\left(u_{c}\right)=2 M(c), \quad \text { by }(\mathrm{C} 2),
\end{aligned}
$$

and so $\xi_{c} \leqslant 2 M(c) / c^{2}<0$. Let $\lambda_{c}=\xi_{c}{ }^{-1}$. Then $c^{2} / 2 M(c) \leqslant \lambda_{c}<0$ and, as in the proof of Lemma 4.3, we then have that

$$
\lambda_{c} \leqslant-K^{2 /(\beta-2)} c^{2(\alpha+\beta-2) /(\beta-2)} .
$$

This completes the proof of the proposition.

THEOREM 4.5. Let the conditions $(\mathrm{C} 0),(\mathrm{C} 1),(\mathrm{C} 2)$, and $(\mathrm{C} 3)^{*}$ hold. Suppose also that $\varphi: H_{1} \rightarrow \mathbb{R}$ is weakly sequentially continuous.

(a) If there exists $c_{0}>0$ such that $M(\underline{c})<0$ for all $c \in\left(0, c_{0}\right)$, then $-\infty \in B G^{*}$ for equation (4.1).

(b) If $M(c) / c^{2} \rightarrow-\infty$ as $c \rightarrow \infty$, then $0 \in B G^{*}(\infty)$ for equation (4.1).

Proof. These results follow immediately from Proposition 4.4.

\section{The Dirichlet problem with decay}

In this section we discuss bifurcation for the problems (1.3) and (1.4) for $n \geqslant 2$. The results depend upon the value of the constant $\sigma>0$ and on the behaviour of the function $r$. The following notation will help us to distinguish the different properties of $r$ which will be used.

(R1) The function $r: \mathbb{R}^{n} \rightarrow[0, \infty)$ is baunded and measurable. Let $0 \leqslant r(x) \leqslant R$ for all $x \in \mathbb{R}^{n}$.

(R2) We let $r(x) \rightarrow 0$ as $|x| \rightarrow \infty$, where $|x|=\left(\sum_{i=1}^{n} x_{i}^{2}\right)^{\frac{1}{2}}$ and $x=\left(x_{1}, \ldots, x_{n}\right) \in \mathbb{R}^{n}$.

(R3) There exist $A>0$ and $t \in(0,2)$ such that $r(x) \geqslant A(1+|x|)^{-t}$ for all $x \in \mathbb{R}^{n}$.

(R4) There exist $B>0$ and $\tau \in(0,2)$ such that $0 \leqslant r(x) \leqslant B(1+|x|)^{-\tau}$ for all $x \in \mathbb{R}^{n}$.

(R5) There exists $\eta>0$ such that $\int_{|x|<\eta} r(x) d x>0$.

In order to use the general formulation introduced in $\S \S 2$ to 4 , we set

$$
\begin{aligned}
& H=L^{2}\left(\mathbb{R}^{n}\right) \text { with }\|u\|=\left\{\int_{\mathbb{R}^{n}} u(x)^{2} d x\right\}^{\ddagger}, \\
& \mathscr{D}(S)=\left\{u \in H: \partial_{i} \partial_{j} u \in H \text { for } 1 \leqslant i, j \leqslant n\right\}
\end{aligned}
$$


and

$$
S u=-\Delta u \equiv-\sum_{i=1}^{n} \partial_{i}{ }^{2} u \text { for } u \in \mathscr{D}(S) .
$$

Then $S: \mathscr{D}(S) \subset H \rightarrow H$ satisfies the condition $(\mathrm{C} 0)$ and $H_{2}$ coincides (up to equivalence of norms) with the Sobolev space which is usually denoted by $W_{2}^{2}\left(\mathbb{R}^{n}\right)$. Furthermore, if we choose $T=S^{\frac{1}{2}}$, the Plancherel extension of the Fourier transform shows that $H_{1}$ coincides with the Sobolev space $W_{2}^{1}\left(\mathbb{R}^{n}\right)$. In particular, for $u \in \mathscr{D}\left(S^{\frac{1}{2}}\right)=H_{1}$,

$$
\|T u\|^{2}=\left\|S^{\frac{1}{2}} u\right\|^{2}=\sum_{i=1}^{n}\left\|\partial_{i} u\right\|^{2}=\||\nabla u|\|^{2}
$$

where $\nabla u=\left(\partial_{1} u, \ldots, \partial_{n} u\right)$. (See [13] for details.)

Let us now consider the non-linear term in (1.3) and (1.4). For $\sigma>0$ and a function $r$ satisfying (R1), we set

$$
F(u)=r|u|^{\sigma} u
$$

and

$$
\varphi(u)=\frac{1}{(\sigma+2)} \int_{\mathbf{R}^{n}} r(x)|u(x)|^{\sigma+2} d x .
$$

With these conventions, we see that the notions of generalized and strong solution introduced in $\$ \S 3$ and 4 correspond to the usual notions of generalized and strong solution of (1.3) and (1.4) used in the theory of partial differential equations.

In the following results we often encounter expressions of the form $a /(n-2)$ where $a>0$. In the case where $n=2$ such expressions are interpreted as $+\infty$.

Lemma 5.1. Suppose that $r$ satisfies the condition $(\mathrm{R} 1)$ and that $\sigma \in(0,4 /(n-2))$. Then $F$ maps $H_{1}\left(=W_{2}^{1}\left(\mathbb{R}^{n}\right)\right)$ continuously and boundedly into $H_{1}^{*}$. Furthermore, $\varphi: H_{1} \rightarrow \mathbb{R}$ is continuously Fréchet differentiable and $\varphi^{\prime}(u) v=\langle F(u), v\rangle$ for all $u, v \in H_{1}$. If $\sigma \in(0,2 /(n-2))$, then $F$ maps $H_{1}$ continuously and boundedly into $H\left(=L^{2}\left(\mathbb{R}^{\prime \prime}\right)\right)$.

Proof. We recall that $H_{1}$ is continuously embedded in $L^{p}\left(\mathbb{R}^{n}\right)$ for all $p \in[2,2 n /(n-2))$ and consequently $L^{p /(p-1)}(\mathbb{R})$ is continuously embedded in $H_{1}^{*}$. Hence $F$ will map $H_{1}$ continuously and boundedly into $H_{1}^{*}$ provided that it maps $L^{p}\left(\mathbb{R}^{n}\right)$ continuously and boundedly into $L^{p /(p-1)}\left(\mathbb{R}^{n}\right)$ for some $p \in[2,2 n /(n-2))$. Now by the basic result on Nemytskii operators (see [3], for example) $F$ maps $L^{p}\left(\mathbb{R}^{n}\right.$ ) continuously and boundedly into $L^{q}\left(\mathbb{R}^{n}\right)$ provided that $\sigma+1=p / q$. Hence $F$ maps $H_{1}$ continuously and boundedly into $H_{1}^{*}$ provided that $\sigma+1=p-1$ for some $p \in[2,2 n /(n-2))$. This is ensured by the requirement that $\sigma \in(0,4 /(n-2))$.

In the same way we see that $F$ maps $H_{1}$ continuously and boundedly into $H$ provided that $\sigma+1=\frac{1}{2} p$ for some $p \in[2,2 n /(n-2))$. This is ensured by the requirement that $\sigma \in(0,2 /(n-2))$.

Finally, we consider the differentiability of $\varphi$. For $u, v \in H_{1}$, we have $F(u) \in L^{p /(p-1)}\left(\mathbb{R}^{n}\right)$ and $v \in L^{p}\left(\mathbb{R}^{n}\right)$, where $p \equiv \sigma+2 \in[2,2 n /(n-2))$. Hence

$$
\langle F(u), v\rangle=\int_{\mathbf{R}^{n}} r(x)|u(x)|^{\sigma} u(x) v(x) d x
$$


and

$$
|\langle F(u), v\rangle| \leqslant\|F(u)\|_{\left.L^{p / p}-1\right)\left(R^{n}\right)}\|v\|_{L^{p}\left(\mathbf{R}^{n}\right)}
$$

where $p=\sigma+2$. In particular, $\varphi(u)=(\sigma+2)^{-1}\langle F(u), u\rangle$ maps $H_{1}$ into $[0, \infty)$. Furthermore, for $u, v \in H_{1}$,

$$
\begin{aligned}
|\varphi(u+v)-\varphi(u)-\langle F(u), v\rangle| \\
=\left|\int_{\mathbb{R}^{n}} r(x)\left\{\int_{0}^{1} \frac{d}{d t} \frac{|u(x)+t v(x)|^{\sigma+2}}{(\sigma+2)} d t-|u(x)|^{\sigma} u(x) v(x)\right\} d x\right| \\
=\left|\int_{\mathbb{R}^{n}} r(x) \int_{0}^{1}\left\{|u(x)+t v(x)|^{\sigma}[u(x)+t v(x)]-|u(x)|^{\sigma} u(x)\right\} d t v(x) d x\right| \\
\quad \leqslant \int_{0}^{1}\|F(u+t v)-F(u)\|_{L^{p(p-1)\left(R^{n}\right)}\|v\|_{L^{p}\left(\mathbb{R}^{n}\right)} d t,}
\end{aligned}
$$

where $p=\sigma+2 \in[2,2 n /(n-2))$. But for $p \in[2,2 n /(n-2))$, there exists a constant $D>0$ (depending only on $p$ and $n$ ) such that $\|v\|_{L^{p}\left(R^{n}\right)} \leqslant D\|v\|_{1}$ for all $v \in H_{1}$. (See (5.1).) Hence

$$
|\varphi(u+t v)-\varphi(u)-\langle F(u), v\rangle| \leqslant D\|v\|_{1} \max _{0 \leqslant 1 \leqslant 1}\|F(u+t v)-F(u)\|_{L^{p / p-1)}\left(\mathbf{R}^{n}\right)},
$$

where $p=\sigma+2$. Since we have already shown that $F$ maps $H_{1}$ continuously into $L^{p /(p-1)}\left(\mathbb{R}^{n}\right)$ it follows that $\varphi$ is differentiable in the sense of Fréchet at $u$ and that $\varphi^{\prime}(u) v=\langle F(u), v\rangle$. This completes the proof of the lemma.

Lemma 5.2. Suppose that $r$ satisfies the conditions (R1) and (R2) and that $\sigma \in(0,4 /(n-2))$. Then $\varphi: H_{1} \rightarrow \mathbb{R}$ is weakly sequentially continuous.

Proof. Let the sequence $\left\{u_{k}\right\}$ converge weakly in $H_{1}$ to $u$. Then there exists a constant $M>0$ such that

$$
\left\|u_{k}\right\|_{1} \leqslant M \quad \text { and }\|u\|_{1} \leqslant M \quad \text { for all } k \in \mathbb{N} .
$$

For $c>0$,

$$
\begin{aligned}
(\sigma+2)\left|\varphi\left(u_{k}\right)-\varphi(u)\right| \leqslant & \left|\int_{|x| \leqslant c} r(x)\left\{\left|u_{k}(x)\right|^{\sigma+2}-|u(x)|^{\sigma+2}\right\} d x\right| \\
& +\int_{|x|>c} r(x)\left|u_{k}(x)\right|^{\sigma+2} d x+\int_{|x|>c} r(x)|u(x)|^{\sigma+2} d x .
\end{aligned}
$$

Now, by the compactness of the Sobolev embeddings on bounded domains, we have that $\left\|u_{k}-u\right\|_{L^{p(B(c))}} \rightarrow 0$ as $k \rightarrow \infty$ for $p \in[2,2 n /(n-2))$, where $B(c)=\left\{x \in \mathbb{R}^{n}:|x| \leqslant c\right\}$. But

$$
\begin{aligned}
& \left|\int_{|x| \leqslant c} r(x)\left\{\left|u_{k}(x)\right|^{\sigma+2}-|u(x)|^{\sigma+2}\right\} d x\right| \\
& \quad=\left|\int_{|x| \leqslant c}\left\{F\left(u_{k}\right)(x) u_{k}(x)-F(u)(x) u(x)\right\} d x\right| \\
& \quad \leqslant\left\|F\left(u_{k}\right)-F(u)\right\|_{L^{q}(B(c))}\left\|u_{k}\right\|_{L^{p}(B(c))}+\|F(u)\|_{L^{q}(B(c))}\left\|u_{k}-u\right\|_{L^{p}(B(c))},
\end{aligned}
$$


where $p=\sigma+2$ and $q=p /(p-1)$. But, as in the proof of Lemma 5.1, $F$ maps $L^{p}(B(c))$ continuously and boundedly in $L^{q}(B(c))$ where $p=\sigma+2$ and $q=p /(p-1)$. Thus we see that

$$
\left|\int_{|x| \leqslant c} r(x)\left\{\left|u_{k}(x)\right|^{\sigma+2}-|u(x)|^{\sigma+2}\right\} d x\right| \rightarrow 0 \quad \text { as } k \rightarrow \infty,
$$

and consequently

$$
\limsup _{k \rightarrow \infty}(\sigma+2)\left|\varphi\left(u_{k}\right)-\varphi(u)\right| \leqslant \sup _{k \in N} \int_{|x|>c} r(x)\left\{\left|u_{k}(x)\right|^{\sigma+2}+|u(x)|^{\sigma+2}\right\} d x .
$$

Let $\varepsilon>0$. Then, by (R2), there exists $c(\varepsilon)>0$ such that $0 \leqslant r(x)<\varepsilon$ for $|x|>c(\varepsilon)$. Hence

$$
\begin{aligned}
\limsup _{k \rightarrow \infty}(\sigma+2)\left|\varphi\left(u_{k}\right)-\varphi(u)\right| & \leqslant \varepsilon \sup _{k \in \mathbb{N}} \int_{|x|>c(\varepsilon)}\left\{\left|u_{k}(x)\right|^{\sigma+2}+|u(x)|^{\sigma+2}\right\} d x \\
& \leqslant \varepsilon\left\{\left\|u_{k}\right\|_{L^{\sigma+2}\left(\mathbf{R}^{n}\right)}^{\sigma+2}+\|u\|_{L^{\sigma+2}\left(\mathbf{R}^{n}\right)}^{\sigma+2}\right\} \\
& \leqslant 2 \varepsilon(D M)^{\sigma+2},
\end{aligned}
$$

by the Sobolev embedding, since $\sigma+2 \in[2,2 n /(n-2))$. This proves that $\varphi\left(u_{k}\right) \rightarrow \varphi(u)$ as $k \rightarrow \infty$.

In order to verify the conditions (C3) or (C3)* in the present context, we begin by recalling the special multiplicative form of the Sobolev inequalities due to Gagliardo, Nirenberg, and Golovkin. (See [5, Theorem 2.2] or [13].) For $p \in[2,2 n /(n-2))$, there exists a constant $D(p, n)$ (depending only on $p$ and $n$ ) such that

$$
\|u\|_{L^{p}\left(\mathbf{R}^{n}\right)} \leqslant D(p, n)\||\nabla u|\|^{\gamma}\|u\|^{1-\gamma}
$$

for all $u \in H_{1}\left(=W_{2}^{1}\left(\mathbb{R}^{n}\right)\right)$, where $\gamma=n\left(\frac{1}{2}-p^{-1}\right)$.

Lemma 5.3. Let $\sigma \in(0,4 /(n-2))$. Then there exists a constant $K(\alpha)>0$ such that

$$
|\langle F(u), u\rangle| \leqslant K(\alpha)\|T u\|^{\alpha}\|u\|^{\sigma+2-\alpha} \text { for all } u \in H_{1},
$$

where

(a) $\alpha=\frac{1}{2} n \sigma$ if $r$ satisfies the condition ( $\left.\mathrm{R} 1\right)$,

(b) $\alpha=n\left(\frac{1}{2} \sigma+s^{-1}\right)$ if $r \in L^{s}\left(\mathbb{R}^{n}\right)$ for some $s \in(2 n /(4-\sigma(n-2)), \infty)$,

(c) $\alpha \in\left[\frac{1}{2} n \sigma, \frac{1}{2} n \sigma+\delta\right)$ where $\delta=\min \left\{\tau, \frac{1}{2}(4-\sigma(n-2))\right\}$ if $r$ satisfies the conditions $(\mathrm{R} 1)$ and (R4).

Proof. We recall that, for $u \in H_{1}\left(=W_{2}^{1}\left(\mathbb{R}^{n}\right)\right), T u=S^{\frac{1}{2}} u$ and that $\|T u\|=\||\nabla u|\|$.

(a) For $u \in H_{1}$, we have

$$
|\langle F(u), u\rangle| \leqslant R \int_{R^{n}}|u(x)|^{\sigma+2} d x \leqslant R\left\{D(\sigma+2, n)\|T u\|^{\gamma}\|u\|^{1-\gamma}\right\}^{\sigma+2},
$$

by (5.1) where $\gamma=n\left(\frac{1}{2}-1 /(\sigma+2)\right)$, since $\sigma+2 \in[2,2 n /(n-2))$. Setting

$$
\alpha=\gamma(\sigma+2)=\frac{1}{2} n \sigma \quad \text { and } K(\alpha)=R D(\sigma+2, n)^{\sigma+2} \text {, }
$$

we have the desired inequality.

(b) For $u \in H_{1}$, by Hölder's inequality,

$$
|\langle F(u), u\rangle| \leqslant\|r\|_{L^{s}\left(R^{n}\right)}\left\||u(x)|^{\sigma+2}\right\|_{L^{s / s-1}\left(R^{n}\right)} .
$$


Since $s>2 n /(4-\sigma(n-2)) \geqslant 1$, we have that $s \in(1, \infty)$ and that

$$
q \equiv(\sigma+2) s /(s-1) \in[2,2 n /(n-2)) \text {. }
$$

Hence

$$
|\langle F(u), u\rangle| \leqslant\|r\|_{L^{s}\left(\mathbf{R}^{m}\right)}\|u\|_{L^{q}\left(\mathbf{R}^{m}\right.}^{\sigma+2} \leqslant\|r\|_{L^{s}\left(\mathbf{R}^{m}\right)}\left\{D(q, n)\|T u\|^{\gamma}\|u\|^{1-\gamma}\right\}^{\sigma+2},
$$

by (5.1) where $\gamma=n\left(\frac{1}{2}-q^{-1}\right)$ and $q=(\sigma+2) s /(s-1)$. Setting $\alpha=\gamma(\sigma+2)$ and $K(\alpha)=\|r\| \|_{L^{s}\left(\mathbf{R}^{n}\right)} D(q, n)^{\sigma+2}$, we obtain the desired inequality.

(c) The hypothesis (R4) implies that $r \in L^{s}\left(\mathbb{R}^{n}\right)$ for all $s>n / \tau>1$. Hence the result follows from parts (a) and (b), provided that

$$
\frac{1}{s}<\min \left\{\frac{\tau}{n}, \frac{4-\sigma(n-2)}{2 n}\right\}
$$

REMARK. If $r$ satisfies the conditions (R1) and (R4) and $4 n^{-1}\left(1-\frac{1}{2} \tau\right)<\sigma<4 /(n-2)$, then (c) shows that $F$ satisfies the condition (C3) but for a value of $\alpha>2$. As we have noted elsewhere $[\mathbf{1 0}, \mathbf{1 1}]$ this implies that there is no bifurcation to the left at $\lambda=0$ for equation (1.3). If we make some additional hypotheses about the regularity of $r$, it is possible to show (as in $[10,11]$ for the one-dimensional case) that $B G=\varnothing$ if $\sigma>4 n^{-1}\left(1-\frac{1}{2} \tau\right)$. This indicates that the following result is quite sharp.

THEOREM 5.4. Suppose that $r$ satisfies the conditions (R1) and (R2) and that $0<\sigma<4 /(n-2)$. Then, for the equation (1.3), we have the following results.

(a) If $r$ satisfies (R3), then $0 \in B G$ provided that $0<\sigma<2 n^{-1}(2-t)$.

(b) If $r$ satisfies (R5), then $-\infty \in B G(\infty)$ provided that $0<\sigma<4 n^{-1}$.

(c) If $n \geqslant 3$ and $r$ satisfies (R3), then $0 \in B G^{*}$ provided that $0<\sigma<2(n-2)^{-1}(2-t)$.

(d) If $n=2$ and $r$ satisfies (R3) and (R4), then $0 \in B G^{*}$.

Proof. By Lemmas 5.1 and 5.2, we have that the conditions (C0), (C1), and (C2) are satisfied in all cases and that $\varphi: H_{1} \rightarrow \mathbb{B}$ is weakly sequentially continuous.

(a) We shall apply Theorem 3.3(a). By Lemma 5.3(a), we have that the condition (C3) is satisfied. Let $c>0$. We shall show that

$$
m(c) \leqslant \frac{1}{2} c^{2} \alpha D(n)-c^{\sigma+2} I(n) \alpha^{\frac{1}{4} n \sigma+\frac{1}{2} t},
$$

for all $\alpha \in(0,1]$, where

$$
\begin{aligned}
D(n) & =\frac{4}{d(n)^{2}} \int_{\mathbf{R}^{n}}|y|^{2} e^{-2|y|^{2}} d y, \\
d(n)^{2} & =\int_{\mathbf{R}^{n}} e^{-2|y|^{2}} d y
\end{aligned}
$$

and

$$
I(n)=\frac{A 2^{-t}}{(\sigma+2) d(n)^{\sigma+2}} \int_{|y| \geqslant 1}|y|^{-t} e^{-(\sigma+2)|y|^{2}} d x,
$$

$A$ and $t$ being the constants appearing in (R3). For $\alpha>0$, let $w_{\alpha}: \mathbb{R}^{n} \rightarrow \mathbb{R}$ be defined by

$$
w_{a}(x)=\frac{\alpha^{\frac{1}{2} n} e^{-\alpha|x|^{2}}}{d(n)} \text { for } x \in \mathbb{R}^{n}
$$


Then $w_{\alpha} \in H_{1}$ and $\left\|w_{\alpha}\right\|=1$ for all $\alpha>0$. Furthermore,

$$
\begin{aligned}
\left\|T w_{\alpha}\right\|^{2} & =\int_{\mathbf{R}^{n}} \sum_{i=1}^{n}\left[\partial_{i} w_{\alpha}(x)\right]^{2} d x \\
& =\int_{\mathbf{R}^{n}} \sum_{i=1}^{n}\left[2 \alpha x_{i} w_{\alpha}(x)\right]^{2} d x \\
& =4 \alpha^{2} \int_{\mathbf{R}^{n}}|x|^{2} w_{\alpha}(x)^{2} d x \\
& =\frac{4 \alpha^{2+\frac{1}{2} n}}{d(n)^{2}} \int_{\mathbf{R}^{n}}|x|^{2} e^{-\alpha|x|^{2}} d x=\alpha D(n),
\end{aligned}
$$

by the change of variable $y_{i}=\alpha^{\frac{1}{2}} x_{i}$. Now, for $\alpha>0$,

$$
\begin{aligned}
& \varphi\left(w_{\alpha}\right)=\frac{\alpha^{\operatorname{tn}(\sigma+2)}}{(\sigma+2) d(n)^{\sigma+2}} \int_{\mathbf{R}^{n}} r(x) e^{-\alpha(\sigma+2)|x|^{2}} d x \\
& \geqslant \frac{A \alpha^{t n(\sigma+2)}}{(\sigma+2) d(n)^{\sigma+2}} \int_{|x| \geqslant 1}(2|x|)^{-t} e^{-\alpha(\sigma+2)|x|^{2}} d x \quad \text { (by (R3)) } \\
& =\frac{A 2^{-t} \alpha^{\frac{1}{t n(\sigma+2)}}}{(\sigma+2) d(n)^{\sigma+2}} \int_{|y| \geqslant \alpha^{t}} \alpha^{\frac{1}{t^{t}}}|y|^{-t} e^{-(\sigma+2)|y|^{2}} \alpha^{-\frac{1}{2} n} d y,
\end{aligned}
$$

by the change of variable $y_{i}=\alpha^{\frac{1}{2}} x_{i}$. Hence for $\alpha \in(0,1]$,

$$
\varphi\left(w_{\alpha}\right) \geqslant I(n) \alpha^{\frac{1}{n \sigma o+\frac{1}{2} t}} .
$$

Since $c w_{\alpha} \in S(c)$ for all $c>0$, (5.2) follows from (5.4) and (5.5). Let $\alpha(c, n)=\left[c^{\sigma} I(n) / D(n)\right]^{1 /(1-\gamma)}$, where $\gamma=\frac{1}{4} n \sigma+\frac{1}{2} t$. Note that $0<\gamma<1$. Given $n \geqslant 2$, there exists $c(n)>0$ such that $\alpha(c, n) \leqslant 1$ for all $c \in(0, c(n))$ and so

$$
m(c) \leqslant-\frac{1}{2} c^{\sigma+2} I(n) \alpha(c, n)^{\gamma}<0
$$

for all $c \in(0, c(n))$. This completes the proof of part (a).

(b) We shall apply Theorem 3.3(b). By Lemma 5.3(a), we have that the condition (C3) is satisfied. Hence we need only show that $m(c) / c^{2} \rightarrow-\infty$ as $c \rightarrow \infty$. For $\alpha>0$, let $w_{\alpha}$ be defined by (5.3). Then

$$
\varphi\left(w_{1}\right)=\frac{1}{(\sigma+2) d(n)^{\sigma+2}} \int_{\mathbf{R}^{n}} r(x) e^{-(\sigma+2)|x|^{2}} d x \geqslant J(n),
$$

where

$$
J(n)=\frac{e^{-(\sigma+2) \eta^{2}}}{(\sigma+2) d(n)^{\sigma+2}} \int_{|x| \leqslant \eta} r(x) d x>0, \quad \text { by }(\mathrm{R} 5)
$$

Hence

$$
\psi\left(c w_{1}\right)=\frac{1}{2} c^{2}\left\|T w_{1}\right\|^{2}-c^{\sigma+2} \varphi\left(w_{1}\right) \leqslant \frac{1}{2} c^{2} D(n)-c^{\sigma+2} J(n), \quad \text { by (5.4). }
$$

Since $c w_{1} \in S(c)$ for all $c>0$, this proves that $m(c) / c^{2} \leqslant \frac{1}{2} D(n)-c^{\sigma} J(n)$, from which it follows that $m(c) / c^{2} \rightarrow-\infty$ as $c \rightarrow \infty$. This proves part (b) of the theorem.

(c) We shall apply Theorem 3.5(a). By Lemma 5.3(a), we see that the condition (C3)* is satisfied for $n \geqslant 3$. We must prove that $M(c) / c^{2+\sigma} \rightarrow-\infty$ as $c \rightarrow \infty$. For 
$\alpha>0$, let $\hat{w}_{\alpha}=\{\alpha D(n)\}^{-\frac{1}{2}} w_{\alpha}$, where $w_{\alpha}$ is defined by (5.3). Then $\hat{w}_{\alpha} \in H_{1},\left\|T \hat{w}_{\alpha}\right\|=1$, and $\left\|\hat{w}_{\alpha}\right\|=\{\alpha D(n)\}^{-\frac{1}{2}}$, for all $\alpha>0$. Furthermore,

$$
\varphi\left(\hat{w}_{\alpha}\right)=\{\alpha D(n)\}^{-\frac{1}{2}(\sigma+2)} \varphi\left(w_{\alpha}\right) \geqslant\{\alpha D(n)\}^{-\frac{1}{2}(\sigma+2)} I(n) \alpha^{\frac{1}{2 n \sigma+\frac{1}{2} t}},
$$

for all $\alpha \in(0,1]$, by (5.5). Now $c \hat{w}_{\alpha} \in S^{*}(c)$ for all $\alpha>0$, and so $M(c) \leqslant \psi^{*}\left(c w_{\alpha}\right)$ for all $\alpha>0$, and

$$
\psi^{*}\left(c w_{\alpha}\right) \leqslant \frac{1}{2} c^{2}\{\alpha D(n)\}^{-1}-c^{2+\sigma}\{\alpha D(n)\}^{-\frac{1}{2}(\sigma+2)} I(n) \alpha^{\frac{1}{4} n \sigma+\frac{1}{2} t},
$$

for $\alpha \in(0,1]$. Hence

$$
M(c) / c^{2+\sigma} \leqslant\left\{2 c^{\sigma} \alpha D(n)\right\}^{-1}-\alpha^{t a(n-2)+\frac{1}{2} t-1} D(n)^{-\frac{1}{2}(\sigma+2)} I(n) \text { for } \alpha \in(0,1] .
$$

Let $\hat{\alpha}(c, n)=\left[D(n)^{\frac{1}{2} \sigma} / c^{\sigma} I(n)\right]^{1 /(\gamma+1)}$, where $\gamma=\frac{1}{4} \sigma(n-2)+\frac{1}{2} t-1$. Note that $\gamma+1>0$. Given $n \geqslant 3$, there exists $c(n)>0$ such that $\hat{\alpha}(c, n) \leqslant 1$ for all $c \geqslant c(n)$, and so $M(c) / c^{2+\sigma} \leqslant-\frac{1}{2} \hat{\alpha}(c, n)^{\gamma} D(n)^{-\frac{1}{2}(\sigma+2)} I(n)$ for $c \geqslant c(n)$. Since $\gamma<0$, this proves that $M(c) / c^{2+\sigma} \rightarrow-\infty$ as $c \rightarrow \infty$ and the proof of part (c) is complete.

(d) We shall again apply Theorem 3.5(a). By Lemma 5.3(c), we see that the condition (C3)* is satisfied. As in part (c), we have that $M(c) / c^{2+\sigma} \rightarrow-\infty$ as $c \rightarrow \infty$. This complete the proof of part (d) of the theorem.

THEOREM 5.5. Suppose that $r$ satisfies (R1) and (R2) and that $0<\sigma<4 /(n-2)$. Then for the equation (1.4) we have the following results.

(a) If $r$ satisfies (R3), then $0 \in B G(\infty)$ provided that $0<\sigma<2 n^{-1}(2-t)$.

(b) If $n \geqslant 3$ and $r$ satisfies (R3), then $0 \in B G^{*}(\infty)$ provided that $0<\sigma<2(n-2)^{-1}(2-t)$.

(c) If $n=2$ and $r$ satisfies (R3) and (R4), then $0 \in B G^{*}(\infty)$.

Proof. By Lemmas 5.1 and 5.2, the conditions (C0), (C1), and (C3) are satisfied in all cases. Furthermore, $\varphi: H_{1} \rightarrow \mathbb{R}$ is weakly sequentially continuous.

(a) We shall apply Theorem 4.2(b). By Lemma 5.3(a), we have that condition (C3) is satisfied. Furthermore the inequality (5.2) holds and so, as in the proof of Theorem 5.4(a), we have that there exists $c(n)>0$ such that $m(c)<0$ for all $c \in(0, c(n))$.

(b) We shall apply Theorem 4.5(b). By Lemma 5.3(a), the condition (C3)* is satisfied for $n \geqslant 3$. But, in the proof of Theorem $5.4(\mathrm{c})$, we have shown that $M(c) / c^{2+\sigma} \rightarrow-\infty$ as $c \rightarrow \infty$, and so, a fortiori, $M(c) / c^{2} \rightarrow-\infty$ as $c \rightarrow \infty$.

(c) We again apply Theorem 4.5 (b), noting that Lemma 5.3 (c) shows that the condition $(\mathrm{C} 3)^{*}$ is satisfied.

\section{The Dirichlet problem with radial symmetry}

In this section we continue our discussion of bifurcation for the problems (1.3) and (1.4), but we no longer assume that $r$ satisfies (R2). Hence the functional $\varphi$ may fail to be weakly sequentially continuous on $W_{2}^{1}\left(\mathbb{R}^{n}\right)$. Since the results of $\S \S 3$ and 4 may fail to hold if $\varphi$ is not weakly sequentially continuous (see [10-12]), we must modify our approach. Noting that $\varphi$ is weakly sequentially continuous on the subspace formed by the radially symmetric elements of $W_{2}^{1}\left(\mathbb{R}^{n}\right)$, we may proceed by seeking radially symmetric solutions of (1.3) and (1.4). Of course this means that $r$ must also be radially symmetric.

Let the spaces $H$ and $H_{1}$ and the functions $T, T_{1}$, and $F$ be as in $\S 5$. Let $O(n)$ denote 
the group of all linear mappings, $\Gamma$, of $\mathbb{R}^{n}$ onto itself such that $|\operatorname{det} \Gamma|=1$. We suppose that $r$ is radially symmetric in the following sense:

(R6) $r(\Gamma x)=r(x)$ for all $\Gamma \in O(n)$ and all $x \in \mathbb{R}^{n}$.

We need some properties of the projection of a function onto its radially symmetric part. Let

$$
P u(x)=\frac{1}{\omega(1)} \int_{|s|=1} u(|x| s) d s(1),
$$

where $u: \mathbb{R}^{n} \rightarrow \mathbb{R}, d S(1)$ is the usual Lebesgue surface measure on the unit sphere in $\mathbb{R}^{n}$, and $\omega(1)$ is the total surface area of this sphere.

Lemma 6.1. (a) The formula (6.1) defines an orthogonal projection, $P: H \rightarrow H$, in $H$ with image

$$
\operatorname{Im} \mathrm{P}=\{u \in H: u \circ \Gamma=u \text { for all } \Gamma \in O(n)\} .
$$

(b) For $u \in H_{1}$, we have $P u \in H_{1}$ and

$$
\|T P u\|=\||\nabla P u|\| \leqslant\|P|\nabla u|\| \leqslant\|T u\| .
$$

Hence the formula (6.1) defines a bounded linear operator from $H_{1}$ into $H_{1}$ which will be denoted by $P_{1}: H_{1} \rightarrow H_{1}$. The dual operator, $P_{1}^{\prime}: H_{1}^{*} \rightarrow H_{1}^{*}$, is an extension of $P: H \rightarrow H$. (Recall the identifications agreed upon in $\$ 2$.)

(c) For all $u \in H_{1}, P_{1}^{\prime} T_{1}^{\prime} T_{1} u=T_{1}^{\prime} T_{1} P_{1} u$.

(d) Suppose that $r$ satisfies (R1) and (R6) and that $0<\sigma<4 /(n-2)$. Then, for all $u \in H_{1}, P_{1}^{\prime} F\left(P_{1} u\right)=F\left(P_{1} u\right)$.

\section{Proof. See [13].}

The next result establishes the uniform asymptotic decay of radially symmetric elements in $W_{2}^{1}\left(\mathbb{R}^{n}\right)$ for $n \geqslant 2$. It is this decay which allows us to recover the weak sequential continuity of $\varphi$.

Lemma 6.2. For $n \geqslant 2$, there exists a constant $B(n)$ (depending only on $n$ ) such that, for $u \in H_{1} \cap \operatorname{Im} P$,

$$
|u(x)| \leqslant B(n)|x|^{t^{(1-n)}}\|u\|_{1} \quad \text { for almost all }|x| \geqslant 1 .
$$

Proof. This result is contained in [7] and a different proof is given in [13].

With $F$ and $\varphi$ as defined in $\S 5$, we set

$$
\Phi(u) \equiv \varphi\left(P_{1} u\right) \quad \text { and } \quad G(u)=P_{1}^{\prime} F\left(P_{1} u\right) \quad \text { for } u \in H_{1} .
$$

Lemma 6.3. Suppose that $r$ satsifies (R1) and that $0<\sigma<4 /(n-2)$. Then $\Phi: H_{1} \rightarrow \mathbb{R}$ is continuously Fréchet differentiable and $\Phi^{\prime}(u) v=\langle G(u), v\rangle$ for all $u, v \in H_{1}$. Furthermore, $\Phi: H_{1} \rightarrow \mathbb{B}$ is weakly sequentially continuous and $G$ maps $H_{1}$ continuously and boundedly in $H_{1}^{*}$. 
Proof. By Lemmas 5.1 and 6.1 (b), $\Phi: H_{1} \rightarrow \mathbb{R}$ is continuously Fréchet differentiable and

$$
\begin{aligned}
\Phi^{\prime}(u) v & =\varphi^{\prime}\left(P_{1} u\right) P_{1} v \\
& =\left\langle F\left(P_{1} u\right), P_{1} v\right\rangle \\
& =\left\langle P_{1}^{\prime} F\left(P_{1} u\right), v\right\rangle=\langle G(u), v\rangle
\end{aligned}
$$

for all $u, v \in H_{1}$.

For the weak sequential continuity of $\Phi$, let $\left\{u_{k}\right\}$ be a sequence in $H_{1}$ which converges weakly to $u$. Then there is a constant $M$ such that

$$
\left\|u_{k}\right\|_{1} \leqslant M \text { and }\|u\|_{1} \leqslant M
$$

for all $k \in \mathbb{N}$. Let $v_{k}=P_{1} u_{k}$ and $v \in P_{1} u$. Then by Lemma $6.1(\mathrm{~b}),\left\{v_{k}\right\}$ is a sequence in $H_{1}$ which converges weakly to $v$. Furthermore,

$$
\left\|v_{k}\right\|_{1} \leqslant M \quad \text { and }\|v\|_{1} \leqslant M \text { for all } k \in \mathbb{N} .
$$

Then, for $c>0$,

$$
\begin{aligned}
(\sigma+2)\left|\Phi\left(u_{k}\right)-\Phi(u)\right|= & (\sigma+2)\left|\varphi\left(v_{k}\right)-\varphi(v)\right| \\
\leqslant & \left|\int_{|x| \leqslant c} r(x)\left\{\left|v_{k}(x)\right|^{\sigma+2}-|v(x)|^{\sigma+2}\right\}\right| d x \\
& +\int_{|x|>c} r(x)\left|v_{k}(x)\right|^{\sigma+2} d x+\int_{|x|>c} r(x)|v(x)|^{\sigma+2} d x .
\end{aligned}
$$

Hence, as in Lemma 5.2,

$$
\begin{aligned}
(\sigma+2) \limsup _{k \rightarrow \infty}\left|\Phi\left(u_{k}\right)-\Phi(u)\right| & =(\sigma+2) \limsup _{k \rightarrow \infty}\left|\varphi\left(v_{k}\right)-\varphi(v)\right| \\
& \leqslant \sup _{k \in \mathbb{N}} \int_{|x|>c} r(x)\left\{\left|v_{k}(x)\right|^{\sigma+2}+|v(x)|^{\sigma+2}\right\} d x .
\end{aligned}
$$

But, according to Lemma 6.2 ,

$$
\left|v_{k}(x)\right| \leqslant B(n)|x|^{\frac{1}{2}(1-n)}\left\|v_{k}\right\|_{1} \leqslant B(n) M|x|^{\frac{1}{2}(1-n)}
$$

and

$$
|v(x)| \leqslant B(n) M|x|^{\frac{1}{2}(1-n)}
$$

for all $k \in \mathbb{N}$ and almost all $|x| \geqslant 1$. Hence, for $c \geqslant 1$,

$$
\begin{aligned}
\int_{|x|>c} r(x)\left\{\left|v_{k}(x)\right|^{\sigma+2}\right. & \left.+|v(x)|^{\sigma+2}\right\} d x \\
& \leqslant R\left[B(n) M c^{\left.\frac{1}{\mid(1-n)}\right]^{\sigma}} \int_{|x|>c}\left\{v_{k}(x)^{2}+v(x)^{2}\right\} d x\right. \\
& \leqslant R\left[B(n) M c^{\left.\frac{1}{2(1-n)}\right]^{\sigma} 2 M^{2} .}\right.
\end{aligned}
$$

Hence, for $c \geqslant 1$,

$$
(\sigma+2) \limsup _{k \rightarrow \infty}\left|\Phi\left(u_{k}\right)-\Phi(u)\right| \leqslant 2 M^{2} R\left[B(n) M c^{\frac{1}{2}(1-n)}\right]^{\sigma} .
$$


Since $\sigma>0$ and $n>1$, this proves that

$$
\Phi\left(u_{k}\right) \rightarrow \Phi(u) \quad \text { as } k \rightarrow \infty .
$$

We shall now discuss the bifurcation of radially symmetric solutions of (1.3). The equation (1.4) can be treated similarly. Let

$$
E G R=\left\{(u, \lambda) \in H_{1} \times \mathbb{R}: T_{1}^{\prime} T_{1} u-F(u)=\lambda u, u \in \operatorname{Im} P \text { and } u \neq 0\right\},
$$

where $H_{1}, T_{1}$, and $F$ are as defined in $\S 5$ and $P$ is defined by (6.1). Then we write $\lambda_{0} \in B G R^{*} / B G^{*} R$ if and only if there exists a sequence $\left\{\left(u_{k}, \lambda_{k}\right)\right\}$ in $E G R$ such that $\lambda_{k} \rightarrow \lambda_{0}$ and $\left\|u_{k}\right\| \rightarrow 0 /\left\|T_{1} u_{k}\right\| \rightarrow 0$ as $k \rightarrow \infty$. Similarly for bifurcation from infinity, we write $\lambda_{0} \in B G R(\infty) / B G^{*} R(\infty)$ if and only if there exists a sequence $\left\{\left(u_{k}, \lambda_{k}\right)\right\}$ in $E G R$ such that $\lambda_{k} \rightarrow \lambda_{0}$ and $\left\|u_{k}\right\| \rightarrow \infty /\left\|T_{1} u_{k}\right\| \rightarrow \infty$ as $k \rightarrow \infty$.

THEOREM 6.4. Suppose that $r$ satisfies (R1) and (R6) and that $0<\sigma<4 /(n-2)$. Then we have the following results for equation (1.3).

(a) If $r$ satisfies (R3), then $0 \in B G R$ provided that $0<\sigma<2 n^{-1}(2-t)$.

(b) If $n \geqslant 3$ and $r$ satisfies ( $\mathrm{R} 3)$, then $0 \in B G^{*} R$ provided that $0<\sigma<2(n-2)^{-1}(2-t)$.

Remark. In the important special case where (R1) and (R6) hold and $0<B \leqslant r(x) \leqslant A$ for all $x \in \mathbb{R}^{n}$, the condition ( $\left.\mathrm{R} 3\right)$ is satisfied for all $t \in(0,2)$. Hence, in this case,

and

$$
0 \in B G R \quad \text { if } 0<\sigma<4 / n
$$

$$
0 \in B G^{*} R \text { if } n \geqslant 3 \text { and } 0<\sigma<4 /(n-2) .
$$

The latter result has already been observed in [2] for the case where $r$ is a positive constant.

Proof. (a) We consider the problem

$$
T_{1}^{\prime} T_{1} v-G(v)=v
$$

which is of the form (1.1). By Lemma 6.2, $G$ and $\Phi$ satisfy the conditions (C1) and (C2) of $\S 3$ and it follows from Lemmas 5.3 (a) and 6.1 that the condition (C3) is fulfilled by $G$. Furthermore, $\Phi: H_{1} \rightarrow \mathbb{B}$ is weakly sequentially continuous. To apply Proposition 3.2 to the equation $(6.2)$ we must show that $\tilde{m}(c)<0$, where

$$
\tilde{m}(c)=\inf \left\{\frac{1}{2}\|T v\|^{2}-\Phi(v): v \in S(c)\right\} .
$$

But the functions $w_{\alpha}$ defined by (5.3) are such that $w_{\alpha} \in \operatorname{Im} P$ for all $\alpha>0$ and so $\Phi\left(w_{\alpha}\right)=\varphi\left(w_{\alpha}\right)$ for all $\alpha>0$. Hence $\tilde{m}(c)<0$ for all $c<0$.

Thus, by Proposition 3.2, there exists $\left(v_{c}, \lambda_{c}\right) \in H_{1} \times \mathbb{R}$ such that

$$
\begin{aligned}
& \left\|v_{c}\right\|=c, \quad\left\|T v_{c}\right\| \leqslant\left[K c^{\beta}\right]^{1 /(2-\alpha)}, \\
& \frac{1}{2}\left\|T v_{c}\right\|^{2}-\Phi\left(v_{c}\right)=\tilde{m}(c)<0, \\
& -K^{2 /(2-\alpha)} c^{2(\alpha+\beta-2) /(2-\alpha)} \leqslant \lambda_{c} \leqslant 2 \tilde{m}(c) / c^{2}<0,
\end{aligned}
$$

and

$$
T_{1}^{\prime} T_{1} v_{c}-G\left(v_{c}\right)=\lambda_{c} v_{c}
$$


Let $u_{c}=P_{1} v_{c}$. Then $\left(u_{c}, \lambda_{c}\right) \in H_{1} \times \mathbb{R}$ and $u_{c} \in \operatorname{Im} P$. Also $\left\|u_{c}\right\| \leqslant\left\|v_{c}\right\|=c$ and $\left\|T u_{c}\right\| \leqslant\left\|T v_{c}\right\| \leqslant\left[K c^{\beta}\right]^{1 /(2-\alpha)}$, by Lemma 6.1 . Since $\frac{1}{2}\left\|T v_{c}\right\|^{2}-\Phi\left(v_{c}\right)<0$, we have that $\Phi\left(v_{c}\right)=\varphi\left(u_{c}\right)<0$. This shows that $u_{c} \neq 0$.

Finally we note that

$$
P_{1}^{\prime} T_{1}^{\prime} T_{1} v_{c}-P_{1}^{\prime} G\left(v_{c}\right)=\lambda P_{1}^{\prime} v_{c}
$$

and so $T_{1}^{\prime} T_{1} u_{c}-F\left(u_{c}\right)=\lambda u_{c}$ by Lemma 6.1. Thus $\left(u_{c}, \lambda_{c}\right) \in E G R$ for equation (1.3) and result (a) is proved.

Part (b) is proved in a similar way using Proposition 4.4.

\section{The Dirichlet problem on $(0, \infty)$}

In this section we consider the problem (1.6). In order to use the general results of $\S \S 2$ and 3 , we set

and

$$
\begin{aligned}
& H=L^{2}(0, \infty) \text { with }\|u\|=\left\{\int_{0}^{\infty} u(x)^{2} d s\right\}^{\frac{1}{2}}, \\
& \mathscr{D}(S)=\left\{u \in H: u^{\prime \prime} \in H \text { and } u(0)=0\right\}
\end{aligned}
$$

$$
S u=-u^{\prime \prime} \quad \text { for } u \in \mathscr{D}(S) \text {. }
$$

The prime denotes differentiation in the sense of distributions. It is well known that $S: \mathscr{D}(S) \subset H \rightarrow H$ is a positive self-adjoint operator (i.e. the condition (C0) is satisfied). Let $T: \mathscr{D}(T) \subset H \rightarrow H$ be the closed operator defined as follows:

$$
\mathscr{D}(T)=\left\{u \in H: u^{\prime} \in H \text { and } u(0)=0\right\}
$$

and

$$
T u=-u^{\prime} \quad \text { for } u \in \mathscr{D}(T) .
$$

Then it follows that

$$
\begin{aligned}
& \mathscr{D}\left(T^{*}\right)=\left\{u \in H: u^{\prime} \in H\right\}, \\
& T^{*} u=u^{\prime} \text { for } u \in \mathscr{D}\left(T^{*}\right),
\end{aligned}
$$

and

$$
T^{*} T=S .
$$

In terms of the notation and conventions of $\S 2, H_{1}=\left\{u \in H: u^{\prime} \in H\right.$ and $\left.u(0)=0\right\}$ and $\|u\|_{1}=\left\{\|u\|^{2}+\left\|u^{\prime}\right\|^{2}\right\}^{\frac{1}{2}}$. We recall that, if $u \in H_{1}$, then $u$ can be identified with a continuous function on $[0, \infty)$ and we have the following inequalities:

$$
\begin{gathered}
u(x)^{2}=-2 \int_{x}^{\infty} u(y) u^{\prime}(y) d y \leqslant 2\|u\|\left\|u^{\prime}\right\| \quad \text { for } x \geqslant 0, \\
|u(x)| \leqslant\left|\int_{0}^{x} u^{\prime}(y) d y\right| \leqslant x^{\frac{1}{2}}\left\|u^{\prime}\right\| \text { for } x>0
\end{gathered}
$$

and (Hardy's inequality)

$$
\int_{0}^{\infty} x^{-2} u(x)^{2} d x \leqslant 4\left\|u^{\prime}\right\|^{2}
$$


In discussion of the equation (1.6) the following notation will help us to distinguish various useful properties of the function $r$.

(B1) Let $r:(0, \infty) \rightarrow[0, \infty)$ be a measurable function which is bounded on compact subsets of $(0, \infty)$. Furthermore, $\sup _{x \geqslant 1} r(x)<\infty$ and there exists a constant $\gamma \geqslant 0$ such that $x^{y} r(x) \rightarrow 0$ as $x \rightarrow 0$.

(B2) $r(x) \rightarrow 0$ as $r \rightarrow \infty$.

(B3) There exist $A>0$ and $t \in(0,2)$ such that $r(x) \geqslant A(1+x)^{-t}$ for all $x>0$.

(B4) There exist $B>0$ and $\tau \in[0,2]$ such that $0 \leqslant x^{\tau} r(x) \leqslant B$ for all $x>0$.

For $\sigma>0$ and $r$ a function satisfying (B1), let

$$
F(u)=r|u|^{\sigma} u
$$

and

$$
\varphi(u)=\frac{1}{(\sigma+2)} \int_{0}^{\infty} r(x)|u(x)|^{\sigma+2} d x .
$$

LEMMA 7.1. Suppose that $\sigma>0$ and that $r$ satisfies the condition (B1) with $\gamma=2+\frac{1}{2} \sigma$. Then $F: H_{1} \rightarrow H_{1}^{*}$ is continuous and bounded, $\varphi: H_{1} \rightarrow \mathbb{R}$ is Fréchet differentiable, and $\varphi^{\prime}(u) v=\langle F(u), v\rangle$ for all $u, v \in H_{1}$. If (B1) holds with $\gamma=1+\frac{1}{2} \sigma$, then $F$ maps $H_{1}$ continuously and boundedly into $H$.

Proof. Let $\xi:[0, \infty) \rightarrow \mathbb{R}$ be a smooth increasing function such that

$$
\xi(x)= \begin{cases}x & \text { for } 0 \leqslant x \leqslant 1 \\ 2 & \text { for } x \geqslant 2\end{cases}
$$

Let $u, v, w \in H_{1}$. Then

$$
\begin{aligned}
|\langle F(u)-F(v), w\rangle| & =\left|\int_{0}^{\infty} r(x)\left\{|u(x)|^{\sigma} u(x)-|v(x)|^{\sigma} v(x)\right\} w(x) d x\right| \\
& \leqslant\|\xi F(u)-\xi F(v)\|\left\|\xi^{-1} w\right\| .
\end{aligned}
$$

But, using (7.3), we have that

$$
\begin{aligned}
\left\|\xi^{-1} w\right\|^{2} & =\int_{0}^{1} x^{-2} w(x)^{2} d x+\int_{1}^{\infty} \xi(x)^{-2} w(x)^{2} d x \\
& \leqslant 4\left\|w^{\prime}\right\|^{2}+\|w\|^{2}=4\|w\|_{1}^{2}
\end{aligned}
$$

and so

$$
|\langle F(u)-F(v), w\rangle| \leqslant 2\|\xi F(u)-\xi F(v)\|\|w\|_{1}
$$

for all $w \in H_{1}$.

Now $x^{1+\frac{1}{2} \sigma} \xi(x) r(x) \rightarrow 0$ as $x \rightarrow 0$, by (B1). Thus the mapping $\xi r$ satisfies the conditions (C1) and (C2) of Theorem 3.1 of [10]. (Clearly the differentiability of $r$ required by $(\mathrm{C} 1)$ is not necessary.) Hence, $\xi F(u) \in H$ for all $u \in H_{1}$ and 
$\|\zeta F(u)-\zeta F(v)\| \rightarrow 0$ as $\|u-v\|_{1} \rightarrow 0$. It follows that $F$ maps $H_{1}$ boundedly and continuously in $H_{1}^{*}$.

The differentiability of $\varphi: H_{1} \rightarrow \mathbb{B}$ is now established much as in Lemma 5.1 and we do not give the details. If (B1) holds with $\gamma=1+\frac{1}{2} \sigma$, the boundedness and continuity of $F$ as a mapping from $H_{1}$ into $H$ follow immediately from Theorem 3.1 of [10].

Lemma 7.2. Suppose that $\sigma>0$ and that $r$ satisfies the conditions (B1) and (B2) with $\gamma=2+\frac{1}{2} \sigma$. Then $\varphi: H_{1} \rightarrow \mathbb{R}$ is weakly sequentially continuous.

Proof. Let $\left\{u_{k}\right\}$ be a sequence which converges weakly to $u$ in $H_{1}$. Then there exists a constant $M>0$ such that

$$
\left\|u_{k}\right\|_{1} \leqslant M \text { and }\|u\|_{1} \leqslant M \quad \text { for all } k \in \mathbb{N} .
$$

Choose $\varepsilon<0$. By (B1), there exists $\delta>0$ such that $0 \leqslant x^{2+\frac{1}{2} \sigma} r(x) \leqslant \varepsilon$ for all $x \in(0, \delta)$. Hence

$$
\begin{aligned}
\int_{0}^{\delta} r(x)\left|u_{k}(x)\right|^{2+\sigma} d x & \leqslant \varepsilon \int_{0}^{\delta} x^{-\left(2+\frac{1}{2} \sigma\right)}\left|u_{k}(x)\right|^{2+\sigma} d x \\
& \leqslant \varepsilon \sup _{0<x<\delta}\left\{x^{-\frac{1}{2}}\left|u_{k}(x)\right|\right\}^{\sigma} \int_{0}^{\delta} x^{-2}\left|u_{k}(x)\right|^{2} d x \\
& \leqslant \varepsilon M^{\sigma} 4 M^{2} \quad \text { (by (7.2) and (7.3)) } \\
& =4 \varepsilon M^{2+\sigma} .
\end{aligned}
$$

Similarly, $\int_{0}^{\delta} r(x)|u(x)|^{2+\sigma} d x \leqslant 4 \varepsilon M^{2+\sigma}$. On the other hand, by (B2), there exists $z>0$ such that $0 \leqslant r(x)<\varepsilon$ for all $x \geqslant z$. Hence

$$
\begin{aligned}
\int_{z}^{\infty} r(x)\left|u_{k}(x)\right|^{2+\sigma} d x & \leqslant \varepsilon \sup _{x \geqslant z}\left|u_{k}(x)\right|^{\sigma} \int_{z}^{\infty}\left|u_{k}(x)\right|^{2} d x \\
& \leqslant \varepsilon M^{\sigma} M^{2} \quad(\text { by }(7.1)) \\
& =\varepsilon M^{2+\sigma} .
\end{aligned}
$$

Similarly, $\int_{z}^{\infty} r(x)|u(x)|^{2+\sigma} d x \leqslant \varepsilon M^{2+\sigma}$. Hence

$$
\begin{aligned}
(\sigma+2)\left|\varphi\left(u_{k}\right)-\varphi(u)\right| & \\
\leqslant & \int_{0}^{\delta} r(x)\left|u_{k}(x)\right|^{2+\sigma} d x+\int_{0}^{\delta} r(x)|u(x)|^{2+\sigma} d x \\
& +\left|\int_{\delta}^{z} r(x)\left\{\left|u_{k}(x)\right|^{2+\sigma}-|u(x)|^{2+\sigma}\right\} d x\right| \\
& +\int_{z}^{\infty} r(x)\left|u_{k}(x)\right|^{2+\sigma} d x+\int_{z}^{\infty} r(x)|u(x)|^{2+\sigma} d x \\
\leqslant & 10 \varepsilon M^{2+\sigma}+\left|\int_{\delta}^{z} r(x)\left\{\left|u_{k}(x)\right|^{2+\sigma}-|u(x)|^{2+\sigma}\right\} d x\right| .
\end{aligned}
$$

Now $u_{k} \rightarrow u$ uniformly on $[\delta, z]$ as $k \rightarrow \infty$. Hence

$$
\limsup _{k \rightarrow \infty}(\sigma+2)\left|\varphi\left(u_{k}\right)-\varphi(u)\right| \leqslant 10 \varepsilon M^{2+\sigma}
$$

and the result is proved. 
LEMMA 7.3. Suppose $\sigma>0$ and that $r$ satisfies the conditions (B1), (B2), and (B4), with $\gamma=2+\frac{1}{2} \sigma$. Then, for all $u \in H_{1}$,

$$
0 \leqslant\langle F(u), u\rangle \leqslant 2^{\frac{1}{2} \sigma+\tau} B\left\|u^{\prime}\right\|^{\frac{1}{2} \sigma+\tau}\|u\|^{2+\frac{1}{2} \sigma-\tau} .
$$

Proof. For $u \in H_{1}$,

$$
\begin{aligned}
0 & \leqslant\langle F(u), u\rangle=\int_{0}^{\infty} r(x)|u(x)|^{2+\sigma} d x \\
& \leqslant B \int_{0}^{\infty} x^{-\tau}|u(x)|^{2+\sigma} d x \quad(\text { by (B4)) } \\
& \leqslant B\left[2\left\|u^{\prime}\right\|\|u\|\right]^{\frac{1}{2} \sigma} \int_{0}^{\infty} x^{-\tau} u(x)^{2} d x \quad \text { (by (7.1)). }
\end{aligned}
$$

But, using Hölder's inequality, we have that

$$
\begin{aligned}
\int_{0}^{\infty} x^{-\tau} u(x)^{2} d x & \leqslant\|u\|^{2-\tau}\left\{\int_{0}^{\infty} x^{-2} u(x)^{2} d x\right\}^{\frac{1}{\xi^{\tau}}} \\
& \leqslant\|u\|^{2-\tau}\left\{4\left\|u^{\prime}\right\|^{2}\right\}^{\frac{1}{2} \tau} \quad \text { (by (7.3)) } \\
& =2^{\tau}\left\|u^{\prime}\right\|^{\tau}\|u\|^{2-\tau} .
\end{aligned}
$$

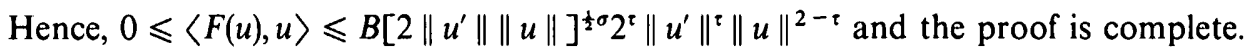

THEOREM 7.4. Suppose that $\sigma \in(0,4)$ and that $r$ satisfies the conditions (B1), (B2), (B3), and (B4) with $\tau \in\left[0,2-\frac{1}{2} \sigma\right)$. Then, for the problem (1.6), we have that $0 \in B G$ provided that $0<\sigma<2(2-t)$.

Proof. Since the condition (B4) holds with $\tau \in\left[0,2-\frac{1}{2} \sigma\right)$, it follows that we can take $\gamma=2+\frac{1}{2} \sigma$ in condition (B1). Hence the condition (C1) of $\$ 2$ is satisfied and $\varphi: H_{1} \rightarrow \mathbb{R}$ is weakly sequentially continuous (Lemmas 7.1 and 7.2). By Lemma 7.3, the conditions (C2) and (C3) are fulfilled. To apply Theorem 3.3 (a) let $c>0$. We shall show that

where

$$
m(c) \leqslant \frac{1}{2} c^{2} \alpha^{2}-c^{2+\sigma} L \alpha^{\frac{1 \sigma}{\sigma}+t} \text { for } \alpha \in(0,1],
$$

$$
L=\frac{2^{2+\sigma-t} A}{(\sigma+2)} \int_{1}^{\infty} y^{2+\sigma-t} e^{-(\sigma+2) y} d y .
$$

For $\alpha>0$, let $v_{\alpha}:[0, \infty) \rightarrow \mathbb{R}$ be the function defined by

$$
v_{\alpha}(x)=2 \alpha^{3 / 2} x e^{-\alpha x} \text { for } x>0 .
$$

Then $v_{\alpha} \in H_{1},\left\|v_{\alpha}\right\|=1$, and $\left\|v_{\alpha}^{\prime}\right\|=\alpha$ for all $\alpha>0$. By (B3),

$$
\begin{aligned}
\varphi\left(v_{\alpha}\right) & =\frac{1}{(\sigma+2)} \int_{0}^{\infty} r(x)\left|v_{\alpha}(x)\right|^{2+\sigma} d x \\
& \geqslant \frac{2^{2+\sigma} A \alpha^{3(2+\sigma) / 2}}{(\sigma+2)} \int_{1}^{\infty}(2 x)^{-t} x^{2+\sigma} e^{-\alpha(\sigma+2) x} d x \\
& =\frac{2^{2+\sigma-t} A \alpha^{\frac{1}{2} \sigma+1}}{(\sigma+2)} \int_{\alpha}^{\infty} y^{2+\sigma-t} e^{-(\sigma+2) y} d y \\
& \geqslant L \alpha^{\frac{1}{2} \alpha+t} \text { for } \alpha \in(0,1] .
\end{aligned}
$$


Hence we have that $c v_{\alpha} \in S(c)$ and

$$
\psi\left(c v_{\alpha}\right) \leqslant \frac{1}{2} c^{2} \alpha^{2}-c^{2+\sigma} L \alpha^{\frac{1}{2} \sigma+t} \text { for } \alpha \in(0,1] .
$$

This proves the inequality (7.4). Let $\hat{\alpha}(c)=\left(c^{\sigma} L\right)^{1 /(2-\gamma)}$ where $\gamma=\frac{1}{2} \sigma+t$. Note that $0<\gamma<2$. Hence there exists $c_{0}>0$ such that $0<\hat{\alpha}(c) \leqslant 1$ for all $c \in\left(0, c_{0}\right)$, and so

$$
m(c) \leqslant-\frac{1}{2} c^{2+\sigma} L \hat{\alpha}(c)^{\gamma}<0 \text { for all } c \in\left(0, c_{0}\right) .
$$

This completes the proof of the theorem.

REMARK. The preceding theorem deals with (1.6) in the case where $r(x) \rightarrow 0$ as $x \rightarrow+\infty$. We note that, for (1.6), there is no counterpart to the results presented in $\S 6$. Indeed, if $r$ satisfies (B1) and is increasing, then $E G=\varnothing$ for all $\sigma>0$ and so there is no bifurcation $[10,11]$. For the problem (1.5) the situation is different and this matter is discussed further in [12].

\title{
References
}

1. V. Benci and D. Fortunato, 'Does bifurcation from the essential spectrum occur?', Comm. Partial Differential Equations, 6 (1981), 249-272.

2. H. BERESTYCKı and P. L. LIONS, 'Existence of stationary states in nonlinear scalar field equations', Bifurcation phenomena in mathematical physics and related topics (ed. C. Bardos and D. Brezis, Reidel, 1980), pp. 269-292.

3. M. A. KraSNOSELSKÜ, Topological methods in the theory of non-linear integral equations (Pergammon, London, 1964).

4. T. KüPPER and D. RIEMER, 'Necessary and sufficient conditions for bifurcation from the continuous spectrum', Nonlinear Anal., 3 (1979), 555-561.

5. O. A. LadyzhensKaya, V. A. Solonnikov, and N. N. URAL'TSEVA, Linear and quasi-linear equations of parabolic type, American Mathematical Society monograph (Providence, R.I., 1968).

6. M. REED and B. SimON, Methods of modern mathematical physics (Academic Press, New York, 1978).

7. W. A. Strauss, 'Existence of solitary waves in higher dimensions', Comm. Math. Phys., 55 (1977), 149162.

8. W. A. Strauss, 'The non-linear Schrödinger equation', Contemporary developments in continumm mechanics and partial differential equations (ed. G. de La Penha and L. A. Medeiros, North Holland, Amsterdam, 1978), pp. 452-465.

9. C. A. Stuart, 'Bifurcation pour des problèmes de Dirichlet et de Neumann sans valeurs propres', $C$. $R$. Acad. Sci. Paris, 288 (1979), 761-764.

10. C. A. Stuart, 'A variational method for bifurcation problems when the linearisation has no eigenvalues', Atti del $3^{\circ}$ S.A.F.A. (1978), Confer. Sem. Mat. Univ. Bari, (1979), 157-180.

11. C. A. StuART, 'A variational approach to bifurcation without eigenvalues', J. Funct. Anal., 38 (1980), 169-187.

12. C. A. StUART, 'Bifurcation for Neumann problems without eigenvalues', J. Differential Equations, 36 (1980), 391-407.

13. C. A. Stuart, 'Bifurcation from the continuous spectrum in the $L^{2}$-theory of elliptic equations in $\mathbb{P}^{n}$ ', Atti del $4^{\circ}$ S.A.F.A. (1980) (Liguori, Naples, 1981), pp. 231-300.

14. K. YosidA, Functional analysis (Springer, Berlin, 1966).

\author{
Département de Mathématiques \\ École Polytechnique Fédérale de Lausanne \\ 61 avenue de Cour \\ CH-1007 Lausanne \\ Switzerland
}

